\title{
Tactile spatial sensitivity and anisotropy
}

\author{
GREGORY O. GIBSON and JAMES C. CRAIG \\ Indiana University, Bloomington, Indiana
}

\begin{abstract}
A gap detection task was examined for its usefulness as a measure of tactile spatial sensitivity and as a measure of anisotropy. In Experiment 1, sensitivity was measured with a gap detection task both with and without a latex glove at three locations on the hand: the fingerpad, fingerbase, and palm. Results showed that sensitivity varied as a function of location and was correlated with changes in the density of innervation of the primary afferent fibers. In accord with other measures of spatial sensitivity, the glove had a moderate effect on sensitivity in the gap detection task. The results both with and without the glove were more similar to those obtained using another measure of spatial sensitivity, the grating orientation task, than to those obtained using the smooth-grooved task, which is considered an intensive measure. In Experiments 2-4, anisotropy was examined using the gap detection and grating orientation tasks, as well as the smooth-grooved task. Locations on the index finger, palm, and arm were tested. Results indicated that anisotropy was revealed only by tasks that relied on spatial cues. The differences between spatial sensitivity measured in the proximal-distal orientation as compared with the lateral-medial orientation varied by location and were as much as $2.35 / 1$. The results are discussed in terms of what they may reveal about the underlying mechanisms responsible for tactile anisotropy.
\end{abstract}

The attempt to develop measures of tactile spatial acuity has a long history. For over 150 years, the standard measure of tactile spatial acuity has been the two-point threshold (Weber, 1834/1996). From the time of its early use, however, researchers have recognized the inherent problems with the two-point threshold as a measure of tactile spatial acuity (Boring, 1942; Friedline, 1918; Tawney, 1895; Weber, 1834/1996; for reviews, see Craig \& Johnson, 2000; Johnson, Van Boven, \& Hsiao, 1994; see also Van Boven \& Johnson, 1994b). More recently, there have been efforts by several researchers to develop new psychophysical measures of tactile spatial acuity (Craig, 1999; Craig \& Kisner, 1998; Craig \& Lyle, 2001; Gibson \& Craig, 2002; Johnson \& Phillips, 1981; Stevens \& Choo, 1996; Stevens \& Patterson, 1995).

One of these new measures that has received considerable attention is the grating orientation (GR/OR) task (Johnson \& Phillips, 1981). In the GR/OR task, subjects discriminate the orientation of a square-wave grating placed on the skin. Performance is measured as a function of the groove width of the grating. It has been shown that performance on the GR/OR task is correlated with the density of innervation of the primary afferent fibers (Gibson

This research was supported by the National Institutes of Health Grant No. DC 00095, National Institute on Deafness and Other Communication Disorders. The authors thank Roger Rhodes for his assistance in these experiments and Dwight Hector for his technical assistance in designing and building the contactors and apparatus used in making the gap detection measurements. They also thank Ranjita Shinde for her assistance in making the figures. Correspondence concerning this article should be addressed to G. O. Gibson, Department of Psychology, Indiana University, Bloomington, IN 47405 (e-mail: gogibson@indiana.edu).
\& Craig, 2002; Van Boven \& Johnson, 1994a, 1994b). In one study that used the GR/OR task, subjects were tested before and after oral surgery that had produced nerve damage. Return to normal performance on the GR/OR task closely paralleled the rate of reinnervation of the primary afferent fibers, as well as the subject's self-report of normal sensory function (Van Boven \& Johnson, 1994b). The GR/OR task has also been used as a measure of spatial acuity at a number of different locations on the skin that are known to vary in their density of innervation (Craig, 1999; Craig \& Kisner, 1998; Craig \& Lyle, 2001; Essock, Krebs, \& Prather, 1997; Gibson \& Craig, 2002; Johnson \& Phillips, 1981; Patel, Essick, \& Kelly, 1997; Sathian \& Zangaladze, 1996; Van Boven \& Johnson, 1994a). In each case, performance on the GR/OR task roughly correlated with changes in density of innervation of the primary afferent fibers. The two types of fibers that convey spatial information are rapidly adapting (RA) and slowly adapting Type I (SAI). Of the two, the SAI fibers are believed to be responsible for the finest spatial information (Johnson \& Hsiao, 1992). The density of the SAI fibers is of more interest in determining spatial acuity.

There are some potential limitations, however, associated with the GR/OR task as a measure of tactile spatial acuity. First, the GR/OR task can be used only on areas of the body that are very sensitive to spatial stimuli (generally the glabrous skin of the hands and lips) and with subjects who have good sensitivity (Patel et al., 1997; Stevens \& Choo, 1996). The difficulty when testing less sensitive subjects or less sensitive areas of the body is that the size of the contactors, relative to the testing site, becomes so large that it is no longer possible to have several cycles of the contactor touching the skin. Another potential problem with the GR/OR task may occur when measuring spatial 
sensitivity at locations on the skin where anisotropy may exist. If spatial sensitivity is considerably greater in one of the two orientations being tested, it may be possible for subjects to differentiate between the two orientations of the contactors using intensive cues rather than spatial cues. For example, if subjects are clearly able to detect the presence of a grating in one orientation but not the other, then they may base their responses on detecting the presence or absence of the grating. Such a discrimination is more likely to be done on the basis of intensive rather than spatial cues (Gibson \& Craig, 2002). The problem of anisotropy and spatial sensitivity is one of the focuses of the present study.

A second task, gap detection (GAP), solves some of the problems associated with the two-point threshold, as well as those noted previously with the GR/OR task (M. B. Jones, Vierck, \& Graham, 1973; Stevens, Alvarez-Reeves, Dipietro, Mack, \& Green, 2003; Stevens \& Choo, 1996; Stevens \& Patterson, 1995). In the GAP task, subjects discriminate between an edge that contains a gap and an edge that contains no gap. Thus, the only salient difference between the edge with no gap and the edge with the gap is the spatial structure of the gap. The GAP task can be used to test spatial sensitivity at different locations on the skin, as well as with subjects of widely different ages and sensitivity (Stevens \& Choo, 1996; Stevens \& Patterson, 1995). Being able to measure spatial sensitivity without having to compare performance at two orientations becomes important on body sites where anisotropy may exist.

The present study had two major aims. The first aim was to examine the usefulness of the GAP task as a measure of spatial acuity (Experiment 1). In Experiment 1, performance on the GAP task was compared with performance on the GR/OR task, as well as on another measure of tactile sensitivity, the smooth-grooved (SM/GV) task. Performance on the SM/GV task is likely mediated by intensive cues and not spatial cues (Gibson \& Craig, 2002). Although previous results with the GAP task have strongly suggested that it was tapping spatial mechanisms (Stevens et al., 2003; Stevens \& Choo, 1996; Stevens \& Patterson, 1995), we wanted to compare it explicitly with these other measures to determine if the GAP task produced results more similar to those of a spatial task (GR/ $\mathrm{OR})$ or an intensive task (SM/GV). The second aim of the study was to examine anisotropy at a number of different sites on the body. In Experiments 2, 3, and 4, three different psychophysical measures of tactile sensitivity (GAP, GR/OR, and SM/GV tasks) were used to examine anisotropy at several locations on the hand and arm. The intent was to compare the amount of anisotropy, if any, that these measures revealed.

A number of psychophysical measures might be used to examine spatial sensitivity. The measures that we used were selected because variations of them had been used in neurophysiological studies and offered the possibility of examining the relationship between the results from such studies and psychophysical results. In addition, the three measures have an obvious orientation component not seen in other measures, such as the error of localization.
Current models of spatial sensitivity hold that a major limiting factor for spatial acuity is the density of innervation of the primary afferent fibers (Johnson \& Hsiao, 1992); however, factors other than the density of innervation may also affect spatial sensitivity. A complete model of spatial sensitivity would need to account for these factors. It has been suggested that one factor affecting spatial sensitivity is the orientation of the stimulus on the body (Greenspan \& Bolanowski, 1996). In early work, researchers observed that sensitivity as measured by the two-point threshold was greater when the two points were oriented across the arm than when the two points were oriented along the arm (anisotropy; Vierordt, 1870; Weber, $1834 / 1996)$. Since the 19th century, however, interest in tactile anisotropy has waned. For example, Weinstein's (1968) oft-cited study on skin sensitivity included no explicit measures of how sensitivity varied as a function of stimulus orientation on the skin. Several recent studies (Essock, Krebs, \& Prather, 1992, 1997; Fuchs \& Brown, 1984; Geldard \& Sherrick, 1983; B. G. Green, 1982; M. B. Jones \& Vierck, 1973; Lechelt, 1988, 1992; Stevens \& Patterson, 1995; Wheat \& Goodwin, 2000; Wong, Ho, $\&$ Ho, 1974) have noted changes in sensitivity as a function of the orientation of the stimuli on the body; however, the only previous systematic studies (Vierordt, 1870; Weber, 1834/1996) of anisotropy, which included multiple test sites, have used the two-point threshold, a problematic measure of spatial sensitivity.

Although there is no single accepted model for tactile anisotropy, a number of mechanisms have been proposed. These include neural mechanisms such as the size and shape of peripheral receptive fields (termed "sensory circles" by Weber, 1834/1996; Stevens \& Patterson, 1995) and orientation-selective neurons in the somatosensory cortex (Essock et al., 1997). Skin ridges have also been proposed as a mechanism (Johnson \& Phillips, 1981; Vega-Bermudez \& Johnson, 2004; Wheat \& Goodwin, 2000): Greater sensitivity at the tip of the finger was observed when grooved contactors were aligned with the dermal ridges rather than perpendicular to the ridges.

The purpose of the current study was to examine a measure of spatial sensitivity, the GAP task (Stevens et al., 2003; Stevens \& Choo, 1996; Stevens \& Patterson, 1995), that could be used on less sensitive areas of the body and that could directly assess anisotropy. In Experiment 1, we compared the results obtained with the GAP task with those obtained previously with the GR/OR and SM/GV tasks. In Experiments 2, 3, and 4, we assessed the suitability of the GAP task for evaluating tactile anisotropy. To do this, we measured tactile anisotropy at several locations with the GAP task, the GR/OR task, and the SM/GV task. The results from the three measures were compared to see what these measures reveal about the nature of tactile anisotropy and the possible mechanisms contributing to tactile anisotropy.

\section{EXPERIMENT 1}

In a recent study, the GR/OR task was used to determine tactile sensitivity on the hand (Gibson \& Craig, 
2002). Measurements were made at three locations on the hand: the index fingertip, proximal fingerpad, and palm. These sites vary in sensitivity (Craig, 1999; Craig \& Lyle, 2001) and in the density of innervation of the primary afferent fibers (Darian-Smith \& Kenins, 1980; Johansson \& Vallbo, 1979). Decreases in the density of innervation result in a corresponding increase in the spacing between receptors as one moves from more distal to more proximal locations on the skin. In the Gibson and Craig (2002) study, we found that the GR/OR threshold varied as a function of location and roughly correlated with the density of innervation. We also measured tactile sensitivity at the same three locations on the hand using a different measure of sensitivity, the SM/GV task. Testing was conducted both with and without a latex glove. The glove was used for two reasons: first, because reductions in tactile sensitivity with gloves may have important applied consequences, and second, because a previous study (Gibson $\&$ Craig, 2002) had shown the presence of this intermediate surface to have a substantial and differential effect on the two measures of sensitivity. Specifically, increases in the GR/OR thresholds with the glove were modest, ranging from $13 \%$ to $36 \%$. In contrast, the $\mathrm{SM} / \mathrm{GV}$ thresholds with the glove increased by $100 \%$ to more than $300 \%$ as compared with the no-glove condition. Without a glove, $\mathrm{SM} / \mathrm{GV}$ thresholds were well below GR/OR thresholds and relatively unaffected by changing the site of stimulation. The results of the GR/OR task were consistent with the view that GR/OR is a valid measure of spatial acuity. The results of the SM/GV task suggested that intensive factors played a major role in performing this task.

In Experiment 1, we measured tactile sensitivity using the GAP task at the same three locations on the hand used in the previous study, testing both with and without a latex glove (Gibson \& Craig, 2002). The results of the current study could then be compared with those obtained in the earlier study. Experiment 1 was designed to test the validity of the GAP task as a measure of spatial acuity comparable to that of the GR/OR task. The question was whether performance on the GAP task paralleled that observed earlier with the GR/OR task (spatial sensitivity) or if it was more similar to that obtained with the SM/GV task (primarily intensive sensitivity).

\section{Method}

Subjects. For all four experiments, the subjects were students at Indiana University who were paid for their participation. For Experiment 1, 6 subjects ( 2 males, 4 females) were tested in the no-glove condition, and 6 additional subjects ( 2 males, 4 females) were tested in the glove condition.

Stimuli. The contactors used in Experiment 1 were similar to those used by Stevens and coworkers (Stevens et al., 2003; Stevens \& Choo, 1996; Stevens \& Patterson, 1995). The contactors were made in pairs. Each pair of contactors was $5 \mathrm{~mm}$ wide and had the same overall length. One contactor was an edge with a gap cut into it, and the other was an edge with no gap (Figure 1). The gap was cut deep enough so that the skin did not touch the bottom of the gap. The gaps were cut such that $5-\mathrm{mm}$ square posts were created at each end of the edge. The gap widths ranged from $0.75 \mathrm{~mm}$ to $12 \mathrm{~mm}$. The overall lengths of the contactors ranged from $10.75 \mathrm{~mm}$ to $22 \mathrm{~mm}$. Table 1 lists the gap widths.

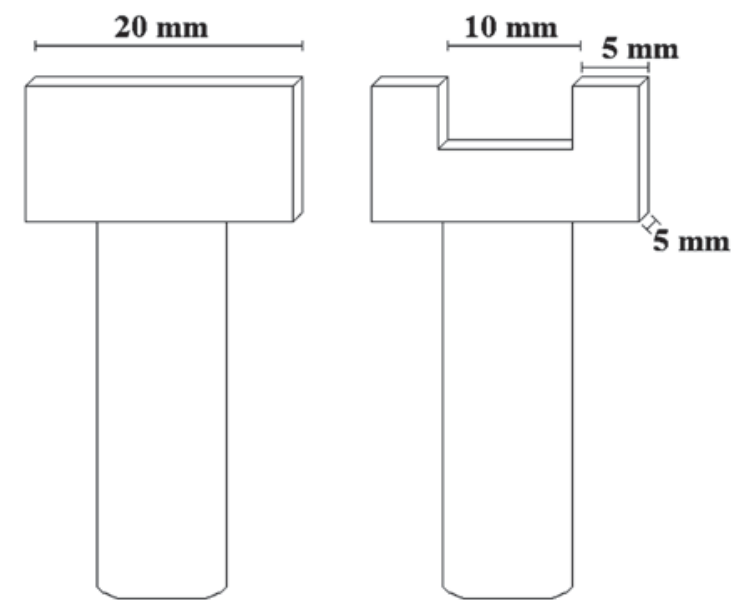

Figure 1. Schematic drawing of one of the contactors used in the gap detection task (10-mm gap contactor). The width of the edge was $5 \mathrm{~mm}$. For the gap contactor, the length of the gap was such that 5-mm square end posts were created. The overall length of the edge was the length of the gap and the length of the end posts.

Apparatus. A spring-handle device was used so that the stimulus could be lowered onto the arm with a force of $100 \mathrm{~g}$. The surgical gloves used in the study were "The Original Perry Style 42 White Latex Surgical Glove" manufactured by Ansell Perry, Inc. (Massilon, $\mathrm{OH}$ ). The surgical gloves come in fitted sizes ranging from $5 \frac{1}{2}$ to 9 . They were $0.20 \mathrm{~mm}$ thick and were the same type as used in an earlier study (Gibson \& Craig, 2002).

Procedure. The subjects were tested individually. In the no-glove condition, each session tested one of three locations. Subjects were tested for four sessions at each location, for a total of 12 sessions. The three locations, shown in Figure 2, were the index fingerpad, fingerbase, and palm. During the testing session, the subject was seated with his or her hand and forearm resting in a prone position on the table; he or she was instructed not to move the hand or fingers during testing. Each trial began with the presentation of the contactor and ended with the subject's response. The contactor remained in contact with the skin until the subject responded (approximately $1-2 \mathrm{sec}$ ). If the subject took longer than $2 \mathrm{sec}$ to respond the contactor was removed and the subject was prompted for a response. During testing, the subjects were asked to keep their eyes closed. The procedures for the glove condition were the same as they were for the no-glove condition with the exception that each subject was tested over three sessions instead of four, and the subject wore a surgical glove on the hand to be tested. Subjects had been fitted with the appropriate-sized gloves that fit snugly without interfering with circulation. There was no indication of slippage between the glove and the surface of the skin.

Subjects were instructed that one of two contactors (an edge without a gap or an edge with a gap) would be presented to the test site. On each trial, subjects were to respond with either "gap" or "no gap." Prior to testing, both contactors were demonstrated while the subject observed the presentation. On the finger, the edge of the contactor was aligned along the proximal-distal axis of the finger. On the palm, the orientation was the same as it was at the sites on the finger (i.e., along the proximal-distal axis of the hand). Each session began with practice trials in which feedback was provided. During testing, however, no feedback was provided. In the no-glove condition, a session consisted of six blocks of 25 trials, for a total of 150 trials per session. For all sessions, testing began with the largest gap width (first block of trials) and proceeded in order to the smallest gap width. The presentation of a gap or no gap on any given trial was determined randomly. 
Table 1

Contactor Sizes (in Millimeters) Used in Each Experiment

\begin{tabular}{|c|c|c|}
\hline \multirow[b]{3}{*}{ Experiment 1} & \multicolumn{2}{|c|}{ Gap Widths for No Glove Versus Glove } \\
\hline & No Glove & Glove \\
\hline & & \\
\hline Fingerpad & $0.75,1,1.25,1.5,2,3$ & $1,1.25,1.5,2,3$ \\
\hline Fingerbase & $1,2,3,4,5,6$ & $2,3,4,5,6$ \\
\hline \multirow[t]{3}{*}{ Palm } & $2,4,6,8,10,12$ & $4,6,8,10,12$ \\
\hline & \multicolumn{2}{|c|}{ Gap Widths for Two Orientations } \\
\hline & Proximal-Distal & Lateral-Medial \\
\hline \multicolumn{3}{|l|}{ Experiment 2} \\
\hline Fingertip & $0.5,0.75,1,1.25,1.5,2,3$ & $0.5,0.75,1,1.25,1.5,2,3$ \\
\hline Fingerpad & $0.5,0.75,1,1.25,1.5,2,3$ & $0.5,0.75,1,1.25,1.5,2,3$ \\
\hline Middle pad & $2,3,4,5,6,8$ & $1.5,2,3,4,5,6$ \\
\hline Fingerbase & $2,3,4,5,6,8$ & $1.5,2,3,4,5,6$ \\
\hline Palm & $3,4,5,6,8,10$ & $2,3,4,5,6,8$ \\
\hline Forearm & $12,14,18,22,26,30$ & $4,6,8,10,14,18$ \\
\hline \multirow[t]{2}{*}{ Upper arm } & $12,14,18,22,26,30$ & $8,10,12,14,18,22$ \\
\hline & \multicolumn{2}{|c|}{ Groove Widths for Three Grating Orientations } \\
\hline \multicolumn{3}{|l|}{ Experiment 3} \\
\hline Fingerpad & \multirow{2}{*}{\multicolumn{2}{|c|}{$\begin{array}{l}0.75,1,1.2,1.5,2,3 \\
2,3,4,5,6\end{array}$}} \\
\hline Fingerbase & & \\
\hline & \multicolumn{2}{|c|}{$\begin{array}{c}\text { Groove Widths for Smooth Versus Grooved } \\
\text { in Two Orientations }\end{array}$} \\
\hline \multicolumn{3}{|l|}{ Experiment 4} \\
\hline Fingertip & \multicolumn{2}{|l|}{$0.35,0.5,0.75,1,1.5,2$} \\
\hline Fingerpad & \multicolumn{2}{|l|}{$0.35,0.5,0.75,1,1.5,2$} \\
\hline Fingerbase & \multicolumn{2}{|l|}{$0.5,0.75,1,1.5,2,3$} \\
\hline Forearm & \multicolumn{2}{|l|}{$1,2,3,4,8,12$} \\
\hline
\end{tabular}

\section{Results and Discussion}

The psychometric functions obtained for the GAP task are shown in Figure 3. The percentage correct is plotted as a function of the log of the gap width. Using nonlinear regression, the data were fitted with a four-parameter sigmoid function (Equation 1), in which two of the parameters $\left(y_{0}=50\right.$ and $\left.a=50\right)$ were constrained so that the functions fit between $50 \%$ and $100 \%$ correct performance:

$$
y=y_{0}+\frac{a}{1+e^{-\left(\frac{x-x_{0}}{b}\right)} .}
$$

In this study, $75 \%$ correct (halfway between chance and $100 \%$ correct) has been adopted as an estimate of threshold (Johnson \& Phillips, 1981). For the results presented in Figure 3, the threshold was calculated using the parameter values and solving Equation 1 for $x$ for a known value of $y(75 \%$ correct). The thresholds for the GAP task with and without gloves, along with the percentage change in sensitivity, are presented in Table 2.

When we compared the results of the GAP detection task with those previously obtained in the GR/OR task (Table 2, no glove), we found that the pattern of results was similar for the two tasks. As expected, without the glove, the GAP thresholds appeared to vary as a function of location in a manner roughly correlated with the density of innervation of the primary afferent fibers, consistent with a measure tapping spatial mechanisms. In general, thresholds were higher with the GAP task; however, there is no a priori reason to expect that the thresholds from the GAP task and the GR/OR task should be the same. The stimuli (gratings and edges) were quite different, as were the tasks. What we did expect, however, was that psychophysical tasks, which are tapping the same underlying neural mechanism, would produce the same relative ordering of thresholds among sites. Indeed, that is

Table 2

Gap Detection, Grating Orientation, and Smooth-Grooved Thresholds (in Millimeters): $\mathbf{7 5 \%}$ Correct Performance and Percentage of Increase in Threshold With Glove

\begin{tabular}{|c|c|c|c|c|c|c|c|c|c|}
\hline & \multicolumn{3}{|c|}{ Gap Detection } & \multicolumn{3}{|c|}{ Grating Orientation } & \multicolumn{3}{|c|}{ Smooth-Grooved } \\
\hline & No Glove & Glove & $\%$ Increase & No Glove & Glove & $\%$ Increase & No Glove & Glove & $\%$ Increase \\
\hline Fingerpad & 1.58 & 1.82 & 15 & 1.24 & 1.69 & 36 & 0.63 & 1.31 & 107 \\
\hline Fingerbase & 5.76 & 6.50 & 13 & 4.35 & 4.93 & 13 & 0.92 & 4.09 & 345 \\
\hline Palm & 4.84 & 8.41 & 74 & 5.73 & 6.98 & 22 & 0.96 & 4.05 & 322 \\
\hline
\end{tabular}

Note-The grating orientation and smooth-grooved thresholds and percent increase data are from Gibson and Craig (2002). 


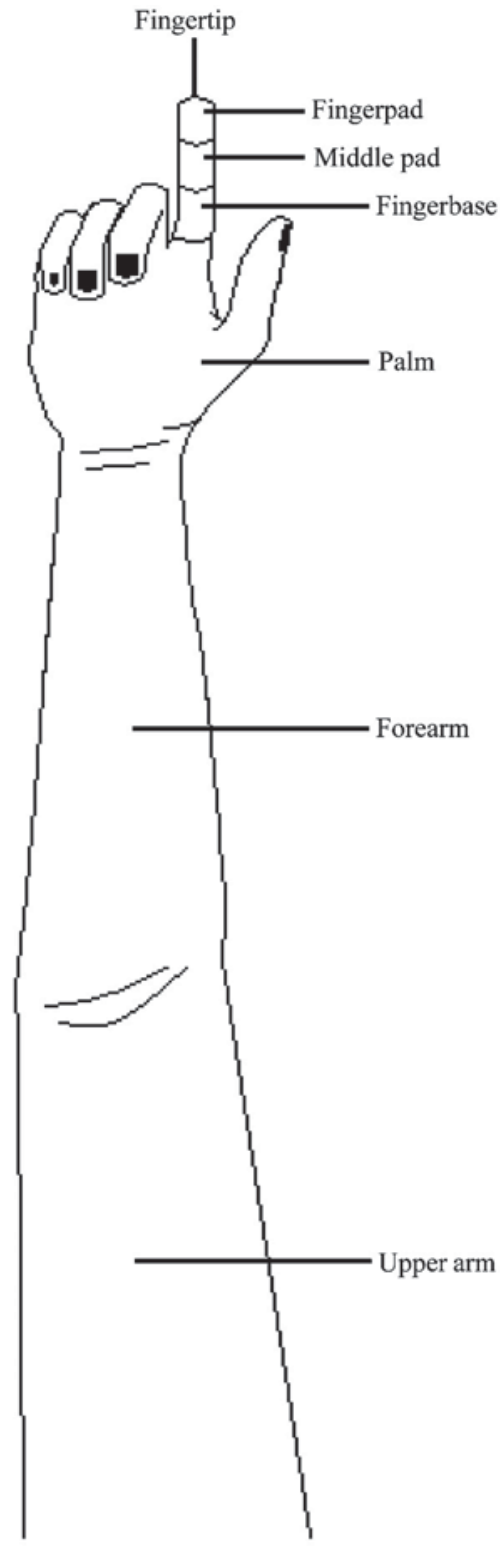

Figure 2. The five test sites on the right index finger and palm were (1) $5 \mathrm{~mm}$ proximal to the fingernail (fingertip); (2) $15 \mathrm{~mm}$ proximal to the fingernail (fingerpad); (3) midway between the two most distal joints (middle pad); (4) midway between the two most proximal joints of the right index finger - that is, the proximal interphalangeal and metacarpophalangeal joints (fingerbase); and (5) centered on the thenar eminence (palm). The test site on the right forearm was midway between the wrist and elbow on the volar surface of the forearm (forearm). On the upper arm the test site was one third of the distance from the elbow to the shoulder on the top of the bicep (upper arm).

what we found with the GAP and GR/OR tasks, with the exception of the palm in the GAP task (no-glove), which had an unexpectedly lower threshold than the fingerbase. A possible reason for this result will be considered in Experiment 2 .
With the glove, increases in the GAP thresholds were relatively uniform and modest, except for the palm, where the increase was $74 \%$. To test whether the glove produced a significant change in threshold, each subject's data were fitted with a sigmoid function (Equation 1) and thresholds were calculated as before. The thresholds were then compared using an independent samples $t$ test. The results indicated that the glove did not have a significant effect on threshold on the fingerpad or fingerbase $[t(10)=-1.00$, $p=.34$, and $t(10)=-1.85, p=.10$, respectively]. On the palm, however, the glove did have a significant effect on threshold $[t(10)=-4.77, p<.01]$.

Next, we compared the changes in performance on the GAP task with those previously obtained with the GR/ OR task (Table 2, glove). We found that the glove had a similar effect on overall performance in both tasks. Again, the exception to this was on the palm. The observed difference between the two conditions (glove, no glove) may be attributable to individual differences because different groups of subjects were tested in the two conditions. In general, the increases in the GAP task thresholds due to the presence of the glove more closely paralleled the increases seen earlier in the GR/OR task but not the SM/GV task (Table 2). Because of this parallel, we concluded that the GAP task is most likely being done using the same spatial cues as in the GR/OR task.

\section{EXPERIMENT 2}

In Experiment 2, the GAP task was used to assess the effect of stimulus orientation (proximal-distal vs. lateralmedial) on sensitivity at several sites on the hand and the arm. We measured spatial anisotropy at five sites on the glabrous skin of the hand (fingertip, fingerpad, middle pad, fingerbase, and palm) and two on the hairy skin of the arm (forearm and upper arm). The two types of skin, glabrous and hairy, have different receptors (Greenspan \& Bolanowski, 1996). Anisotropy has been observed and measured several times on the forearm (Geldard \& Sherrick, 1983; B. G. Green, 1982; Vierordt, 1870; Weber, 1834/ 1996) and fingerpad (Essock et al., 1992, 1997; Lechelt, 1988, 1992; Stevens \& Patterson, 1995; Vierordt, 1870; Weber, 1834/1996; Wheat \& Goodwin, 2000) using a variety of different measures of tactile sensitivity. The remaining experiments in this study focused on a systematic investigation of tactile anisotropy.

\section{Method}

Subjects. Six subjects ( 4 males, 2 females) were tested at each of the hand locations; on the forearm, 5 subjects ( 2 males, 3 females $)$ were tested; and on the upper arm, 8 subjects ( 5 males, 3 females) were tested.

Stimuli. The stimuli used in Experiment 2 were of the same type as those used in Experiment 1.

Apparatus. In testing sites on the hand, a counterweighted lever was used to present the contactors to the skin with 100-g force. An air shock was used to control and smooth the travel of the lever arm to which the contactor was attached. This device was used on the five test sites on the hand. On the arm (forearm and upper arm), the 
Table 3

Gap Detection Thresholds (in Millimeters): 75\% Correct Performance Proximal-Distal Versus Lateral-Medial and Proximal-to-Lateral Ratio

\begin{tabular}{lccc}
\hline & Proximal-Distal & Lateral-Medial & Ratio \\
\hline Fingertip & 1.08 & 1.61 & $0.67: 1$ \\
Fingerpad & 1.70 & 1.63 & $1.04: 1$ \\
Middle pad & 4.00 & 2.64 & $1.52: 1$ \\
Fingerbase & 5.13 & 3.62 & $1.42: 1$ \\
Palm & 7.17 & 5.30 & $1.35: 1$ \\
Forearm & 22.93 & 9.75 & $2.35: 1$ \\
Upper arm & 20.07 & 12.21 & $1.64: 1$ \\
\hline
\end{tabular}

same apparatus that was used in Experiment 1 was used to present the contactors to the skin with 100 -g force.

Procedure. The subjects were tested individually. They were randomly assigned to start each condition with the contactors in either the proximal or the lateral orientation. Thereafter, they alternated sessions between the two orientations. The subjects were tested over six sessions (three in the proximal orientation and three in the lateral) at each of five locations on the hand and two locations on the arm. The locations are shown and labeled in Figure 2.

The testing procedure was similar to that used in Experiment 1. During testing, the subject was seated with his or her right arm extended and resting on the table. On each trial, subjects were presented with either a gap or a no-gap contactor. The edge of the contactor was aligned along either the proximal-distal axis or the lateralmedial axis of the hand. The labeling of the contactors' orientation is in relationship to the length of the edge. This way of designating the orientation of the contactors is in accordance with the way in which previous investigators have specified the orientation when measuring sensitivity with the two-point threshold (Vierordt, 1870; Weber, 1834/1996). The importance of defining the orientation in this manner will be discussed in more detail in Experiment 3.

On the fingertip and fingerpad, a session consisted of seven blocks of 30 trials, for a total of 210 trials per session. As in Experiment 1, testing began with the largest gap width (first block of trials) and proceeded in order to the smallest gap width. The same gap widths were used in both the proximal and lateral conditions at these locations (Table 1). At all the other testing locations, a session consisted of six blocks of 30 trials, for a total of 180 trials per session. Also at these other locations, different ranges of gap widths were required so that the full psychometric function could be captured (Table 1).

\section{Results and Discussion}

The psychometric functions obtained for the gap detection task in the two orientations are shown in Figure 4. The percentage correct is plotted as a function of the log of the gap width. As in Experiment 1, the data were fitted with a four-parameter, sigmoid function (Equation 1). The thresholds were calculated using the same method as in Experiment 1. The thresholds for the GAP task in both orientations, along with the ratio (proximal to lateral) between the two orientations, are presented in Table 3.

For the fingertip and fingerpad, a repeated measures ANOVA was conducted to see whether there was a significant effect of orientation on performance. For the fingertip (see Figure 4), we found significant anisotropy $[F(1,5)=$ $22.88, p<.01]$. At this location, our subjects were about $33 \%$ more sensitive in the proximal orientation than in the lateral orientation. A visual examination of the fingertip shows that the skin ridges are parallel to one another and oriented along the lateral-medial axis of the finger. It is important to note how orientation (proximal-distal vs. lateralmedial) is specified with the GAP task. The necessity of keeping this specification clear becomes apparent in Experiments 3 and 4 when the results from the GR/OR task and the SM/GV task are examined. In the GAP task, the contactor orientation is labeled in reference to the length of the edge. This means that in the proximal-distal orientation, the edge of the gap is oriented parallel to the skin ridges. The significance of the skin ridges will be considered further in the General Discussion section. These results are generally in agreement with an earlier finding by Wheat and Goodwin (2000), who also found greater sensitivity when the edge of the groove (equivalent to the edge of the gap) was parallel with the dermal ridges. For the fingerpad (see Figure 4), we found no significant difference in sensitivity between the two orientations $[F(1,5)=0.83, p=.41]$. The threshold in the proximal orientation was $1.70 \mathrm{~mm}$, and in the lateral orientation, it was $1.63 \mathrm{~mm}$. The ratio in this case was essentially $1: 1$. If anisotropy is due only to neural factors, then, based on these results, we should expect to find a noticeable difference in the neural organization between the fingertip and the fingerpad. From the ratios in Table 3, it appears that at the fingerpad, anisotropy is changing from greater sensitivity in the proximal-distal orientation to greater sensitivity in the lateral-medial orientation.

Because different gap widths were used to test each orientation for the remaining locations, a repeated measures ANOVA was no longer an appropriate statistic to use. As in Experiment 1, each subject's data for the remaining locations were fitted with sigmoid functions (Equation 1) and their $75 \%$ thresholds calculated. The thresholds were compared using a paired samples $t$ test. For the middle pad, the fingerbase, and the palm, we found significant anisotropy in the direction opposite to that observed on the fingertip $[t(5)=-3.39, t(5)=-3.19$, and $t(5)=$ -7.33 , respectively, $p \mathrm{~s}<.05$ ]. The final two testing locations were on the arm. There was significant anisotropy for both the forearm and the upper arm $[t(4)=-10.92$, and $t(7)=-5.78$, respectively, $p \mathrm{~s}<.01]$. On the forearm, the threshold in the lateral orientation was $9.75 \mathrm{~mm}$, and in the proximal orientation, $22.93 \mathrm{~mm}$. The ratio between the two orientations was 2.35:1 (proximal to lateral), the largest difference that we found. On the upper arm, we found less anisotropy than on the forearm (Table 3). The results from the arm are significant for several reasons. First, there are no skin ridges on the arm, yet this is where we found the two largest differences in sensitivity between the two orientations. Second, substantial anisotropy is seen on both hairy and glabrous skin. Third, the large anisotropy on the forearm, which has been noted by other investigators (Geldard \& Sherrick, 1983; B. G. Green, 1982; Vierordt, 1870; Weber, 1834/1996), is not seen on the upper arm, suggesting the result is not simply due to testing hairy skin or less sensitive areas.

As expected, thresholds increase in an orderly fashion as the test site moves from more distal locations to more 


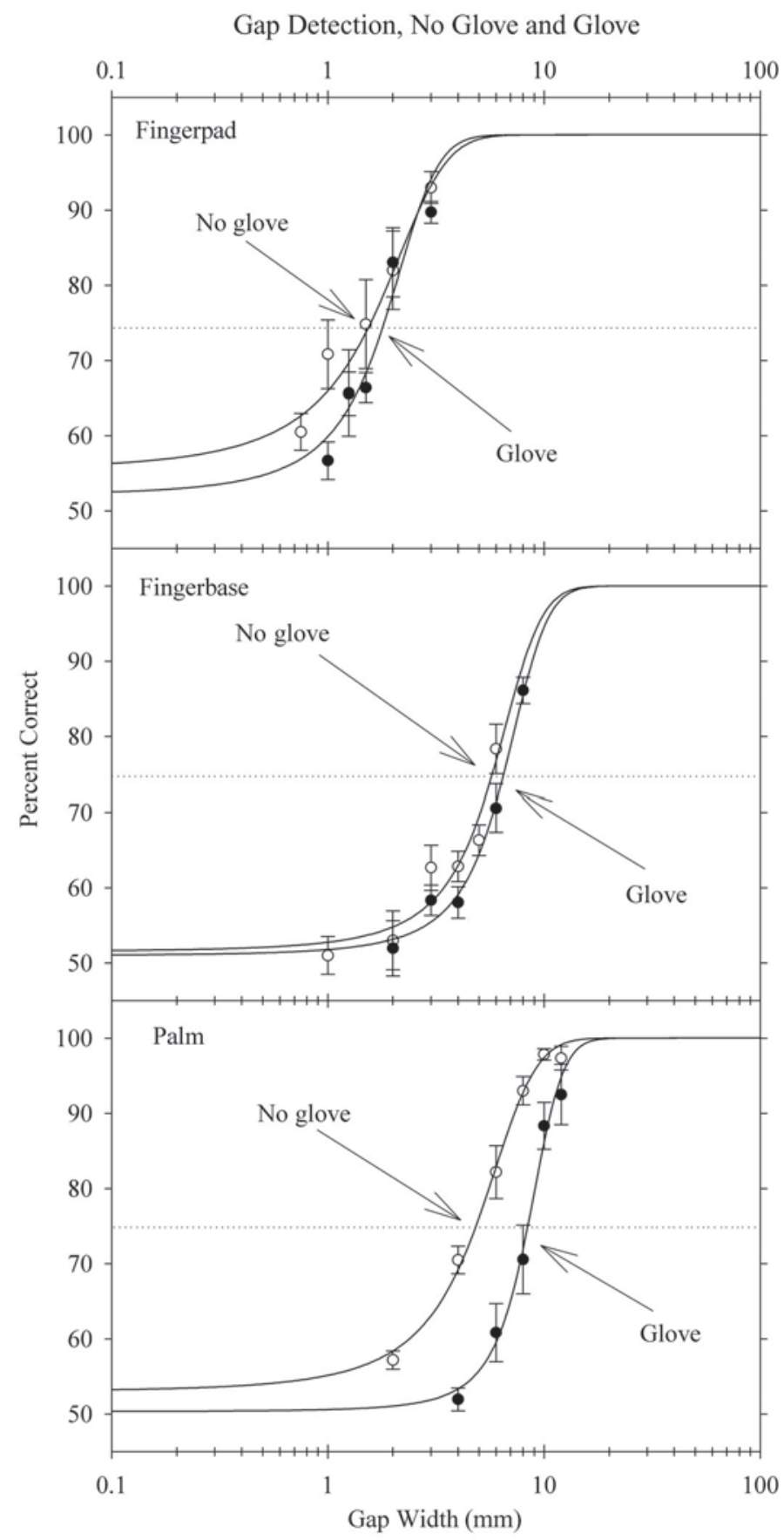

Figure 3. Experiment 1. Percentage correct as a function of the $\log$ gap width (in millimeters) at the fingerpad, fingerbase, and palm, both with and without a glove for the gap detection task. Error bars represent \pm 1 standard error of the mean. The dashed line represents $75 \%$ correct performance.

proximal locations on the hand and arm (Table 3). There was, however, no decline in the overall threshold - that is, the average of the proximal and lateral orientation thresholds, between the forearm and upper arm.

A surprising result from Experiment 1 was that the threshold for the palm $(4.84 \mathrm{~mm})$ was lower than the threshold for the fingerbase $(5.76 \mathrm{~mm})$. In light of the results from Experiment 2 (Table 3), this result appears to be due to the orientations of the contactor on the palm. In Experiment 1, the orientation used on the thenar eminence was in the more sensitive orientation (across the thenar eminence), and on the fingerbase, the orientation 


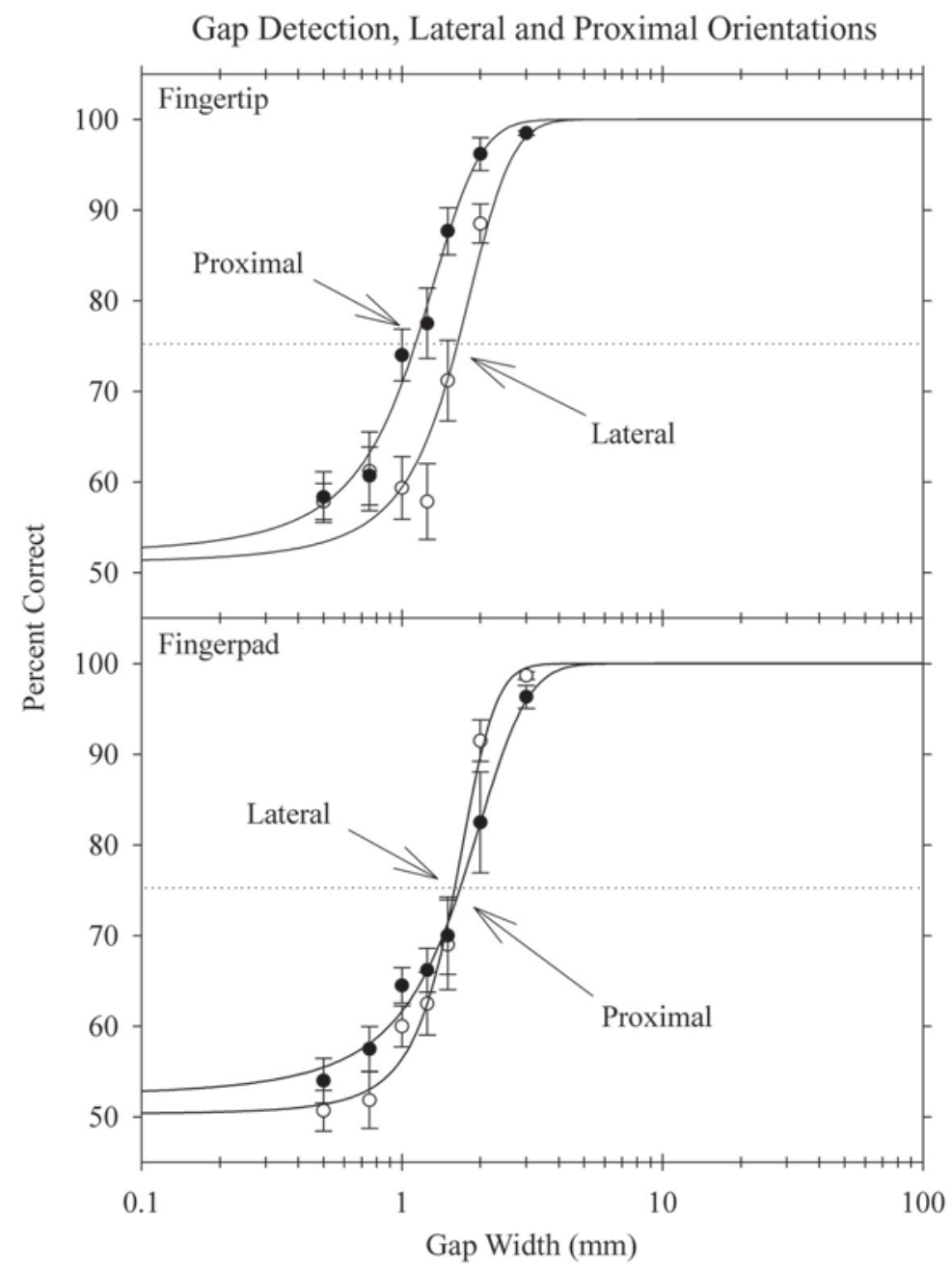

Figure 4. Experiment 2. Percentage correct as a function of the log gap width (in millimeters) at the fingertip, fingerpad, middle fingerpad, fingerbase, palm, forearm, and upper arm for two orientations (proximal and lateral) of the contactor for the gap detection task. Error bars represent \pm 1 standard error of the mean. The dashed line represents $\mathbf{7 5 \%}$ correct performance.

was in the less sensitive orientation (along the finger). When the thresholds are compared on similar orientations (proximal compared with proximal and lateral with lateral) between the two sites, the thenar eminence is, as expected, less sensitive than the fingerbase.

\section{EXPERIMENT 3}

The main aim of Experiment 3 was to obtain measures of sensitivity in the proximal and lateral orientations for the GR/OR task and to compare these results with those obtained from the GAP task. To avoid confusion when describing the orientation of the gratings as proximal-distal or lateral-medial, we will be consistent with the way in which these terms are used with respect to the edges and gaps in the GAP task. When the GAP task is compared with the GR/OR task, the issue of how to specify the orientation of the gratings becomes important (see Appendix). In earlier studies using the GR/OR task (e.g., Vega-Bermudez \& Johnson, 2004; Wheat \& Goodwin, 2000), the alignment of the ridges and grooves was used to specify the orientation. As compared to the way in which orientation has been specified in the present study and in previous studies using the GAP and the twopoint threshold tasks, using this specification of the alignment of the ridges results in a reversal of the terms. For example, a proximal orientation in the previous GR/OR studies is the same as the lateral orientation in the present study, and a lateral orientation is the same as the proximal orientation in the present study. The Appendix goes into more detail concerning the reasons for the differences in terminology.

One of the limitations of the GR/OR task is that it requires using two orientations of the stimulus to obtain 


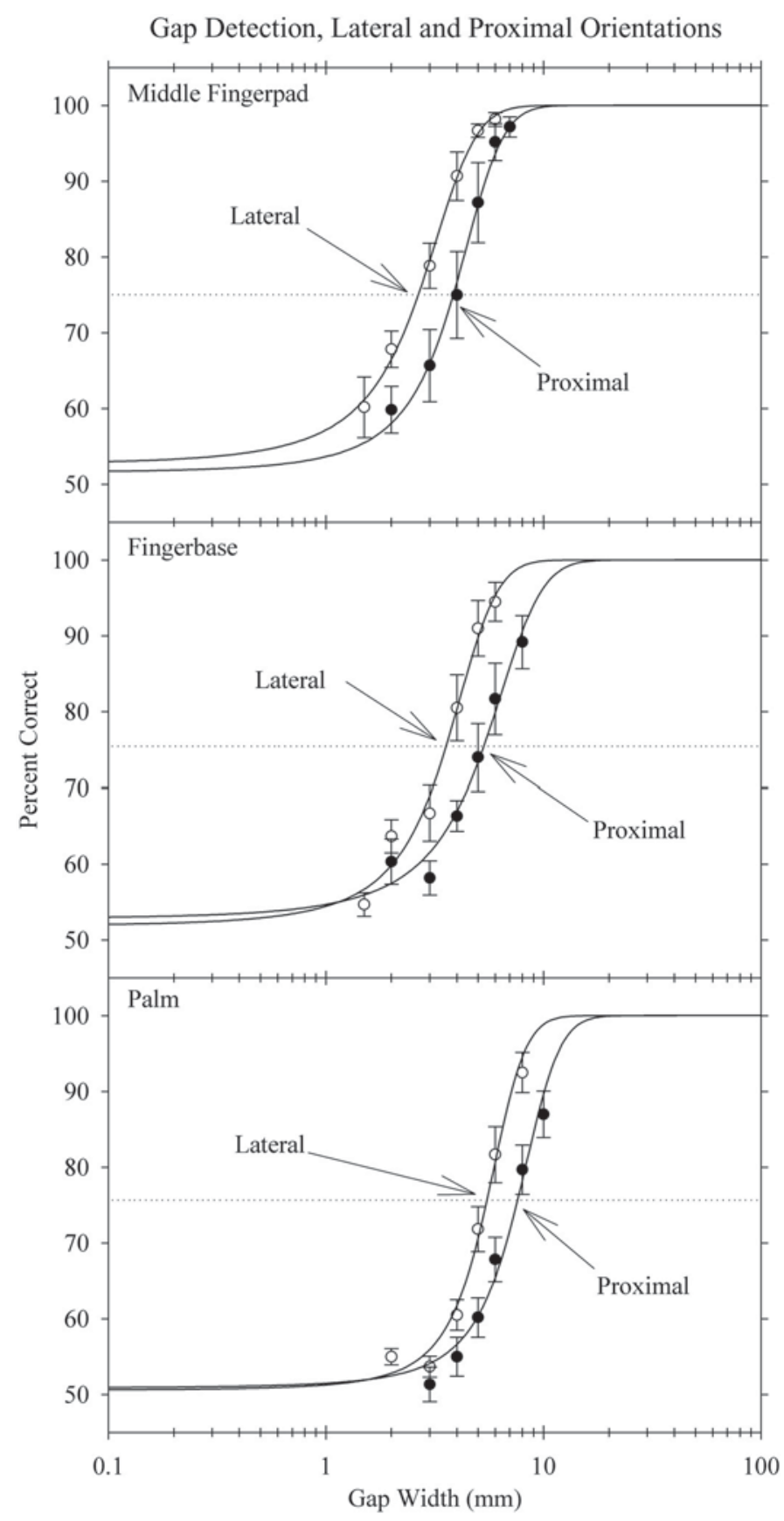

Figure 4 (Continued).

threshold. As noted, in locations where anisotropy exists, subjects may be able to differentiate between the two orientations of the contactors using intensive cues (Gibson \& Craig, 2002). Furthermore, the GR/OR task is limited in its usefulness in studying tactile anisotropy because an independent threshold for each orientation cannot be obtained when only two choices are available (although some types of comparisons are possible; Vega-Bermudez \& Johnson, 2004). The aim of Experiment 3 was to measure spatial sensitivity in the proximal-distal and lateral- medial orientations using the GR/OR task. To do this we used a three-alternative, forced-choice (3AFC) design. The contactor was presented in one of three possible orientations - proximal, lateral, or oblique. By using a 3AFC design, we were able to calculate the performance for each orientation of the contactor independently by determining the hit and false alarm rates. For example, to determine sensitivity in the proximal orientation, a hit was defined as responding "proximal" when the contactor was proximal; a false alarm was defined as responding "proximal" 
Gap Detection, Lateral and Proximal Orientations

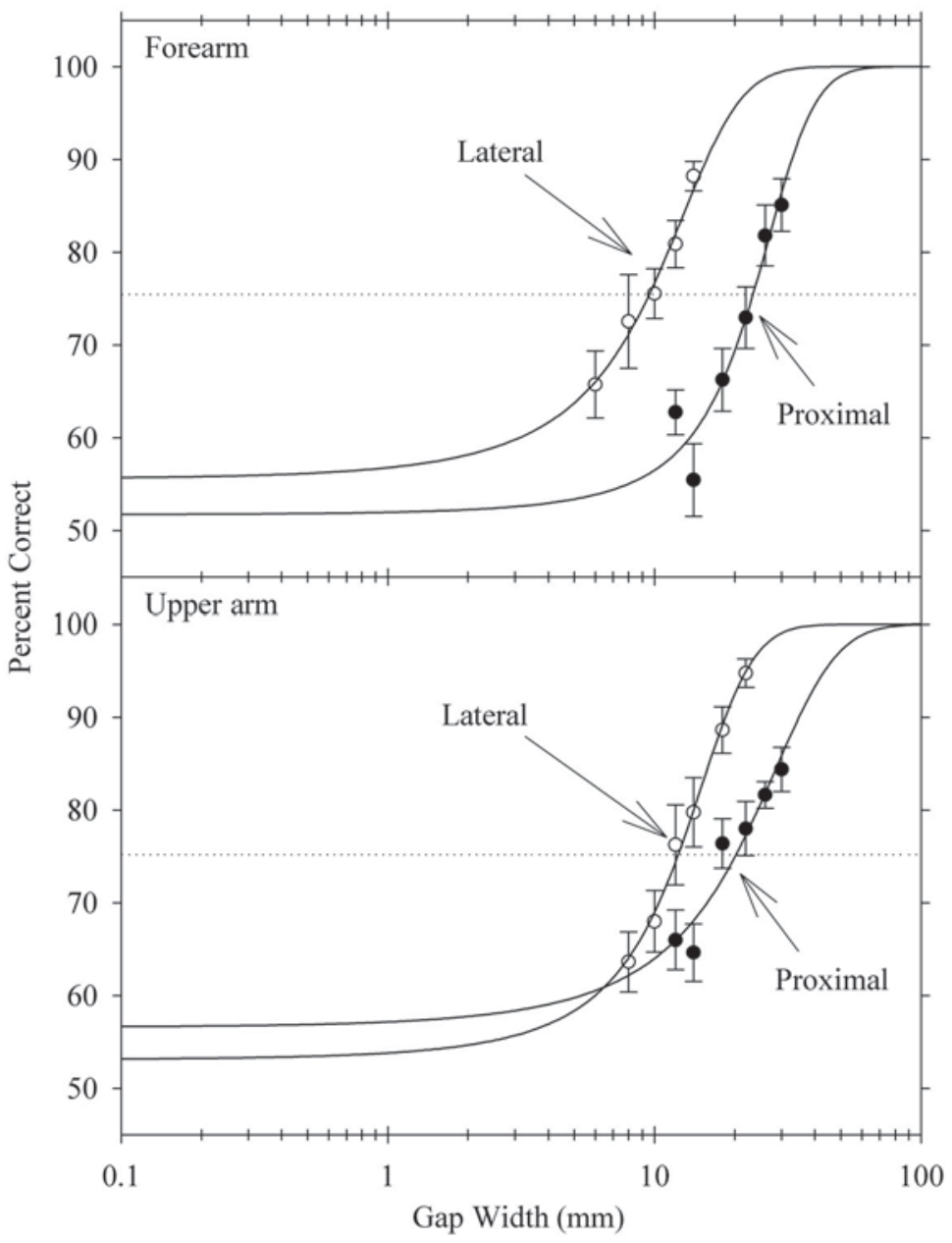

Figure 4 (Continued).

when the contactor was either lateral or oblique. From the hit and false alarm rates, we were able to calculate $P(\mathrm{C})_{\max }$, the proportion of correct responses corrected for response bias (Green \& Swets, 1966), allowing us to determine sensitivity separately for each orientation and compare differences in anisotropy as measured by the GAP task and GR/OR task.

\section{Method}

Subjects. In Experiment 3, 6 subjects were tested ( 2 males, 4 females). These subjects had not been tested in either Experiment 1 or 2 .

Stimuli. The stimuli used were domed contactors with squarewave gratings of equal land/groove widths cut into them. These contactors (JVP Domes) are commercially available through Stoelting Co. (Wood Dale, IL). The grooves in the contactors were cut deep enough so the skin did not touch the bottom of the groove. These contactors were $19 \mathrm{~mm}$ in diameter. Each set consisted of eight commercially made contactors with groove widths ranging from 3.0 to $0.35 \mathrm{~mm}$. In cases where threshold exceeded $3 \mathrm{~mm}$, additional contactors were machined with larger groove widths (Table 1). These additional contactors were also $19 \mathrm{~mm}$ in diameter.
Apparatus. In the GR/OR task, the counterweighted apparatus described in Experiment 2 was used to present the contactors to the test site.

Procedure. The subjects were tested individually. Two locations on the index finger were tested: the fingerpad and the fingerbase. The testing procedures were similar to those used on the hand sites in Experiment 2. The main difference in the procedure was that in Experiment 3, the design was a $3 \mathrm{AFC}$ procedure. The subjects were instructed that a grooved contactor would be presented in one of three orientations: proximal-distal, lateral-medial, or left oblique $\left(45^{\circ}\right.$ to the left of the proximal orientation). The three orientations were shown and explained to the subjects. On each trial, the subjects responded with the orientation of the contactor. Every session began with practice trials in which feedback was provided. During testing, no feedback was provided.

On the fingerpad, a session consisted of six blocks of 30 trials, for a total of 180 trials per session. Each session started with the first block using a groove width of $3 \mathrm{~mm}$. Successive blocks of trials tested smaller groove widths in order (Table 1). On the fingerbase, a session consisted of five blocks of 30 trials, for a total of 150 trials per session. Each session started with the first block using a groove width of $6 \mathrm{~mm}$. Successive blocks of trials tested smaller groove widths in order (Table 1). Subjects were tested for four sessions at each site. 


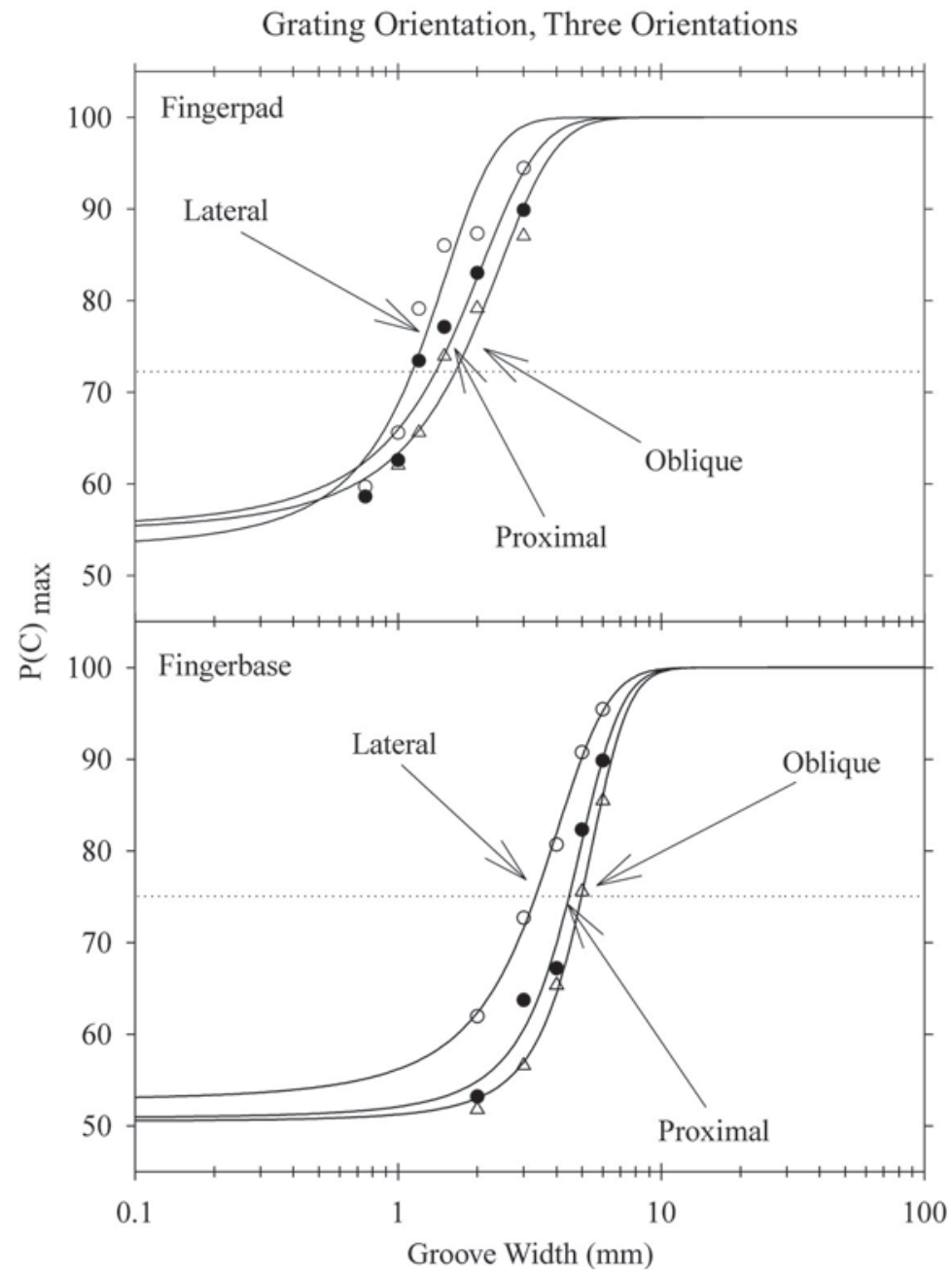

Figure 5. Experiment 3. $P(C)_{\max }$ as a function of the $\log$ of the groove width (in millimeters) at the fingerpad and fingerbase for three orientations (proximal, lateral, and oblique) of the contactor for the grating orientation task. The orientations are defined as indicated in the Appendix. The dashed line represents $\mathbf{7 5 \%}$ correct performance.

\section{Results and Discussion}

The data in Experiment 3 were analyzed by calculating the hit and false alarm rates for each orientation of the contactor. As noted, for the proximal orientation, a hit was defined as responding "proximal" when the contactor was presented in the proximal orientation, and a false alarm was defined as responding "proximal" when the contactor was presented in either one of the other two orientations. In a similar fashion, hits and false alarms were defined for the other two orientations. From the hit and false alarm rates, we calculated $P(\mathrm{C})_{\max }$ for each groove width and orientation of the contactor for each subject.

The psychometric functions obtained for the GR/OR task are shown in Figure 5. Here $P(\mathrm{C})_{\max }$ is plotted as a function of the $\log$ of the groove width for each of the three orientations. As in Experiments 1 and 2, the data were fitted with a four-parameter sigmoid function (Equa- tion 1). From Figure 5, it appears that subjects were most sensitive in the lateral orientation of the grating and least sensitive for the oblique orientations of the grating. For the fingerpad, a factorial ANOVA revealed a significant effect of orientation on performance $[F(2,90)=5.00, p<$ $.05]$. As expected, there was also a significant effect of groove width on performance $[F(5,90)=19.13, p<.05]$; however, there was no significant interaction between orientation and groove width $[F(10,90)=0.004, p=.99]$. Post hoc Bonferroni analysis indicated no significant difference in performance between the lateral and proximal orientations or between the proximal and oblique orientations for the fingerpad ( $p=.23, p=.53$, respectively). There was, however, a significant difference in performance between the lateral and oblique orientations $(p<.05)$. For the fingerbase, there was also a significant effect of orientation and groove width $[F(2,75)=13.71$; 
$F(4,75)=33.85, p \mathrm{~s}<.05$, respectively]. As with the fingerpad, there was no significant interaction $[F(2,90)=$ $0.27, p=.97]$. Also similar to the results from the fingerpad, post hoc Bonferroni tests indicated no significant difference between the proximal and oblique orientations $(p=.18)$. Unlike for the fingerpad, however, for the fingerbase, there was a significant difference in performance between the lateral and proximal orientations as well as lateral and oblique orientations ( $p$ s $<.05)$.

The thresholds, $75 \%$ correct, were calculated using the same method as previously described. The thresholds for the GR/OR task in the three orientations are presented in Table 4. The threshold values reflect what is seen in Figure 5: At both the fingerpad and fingerbase, the most sensitive orientation was the lateral orientation, followed by the proximal and then oblique orientations. The results of the GR/OR task parallel those of the GAP task. For the fingerpad, we found no significant anisotropy between the proximal and lateral orientations. These results are consistent with the conclusions reached in Experiment 2: The fingerpad seems to be a point of transition where anisotropy changes from the proximal being more sensitive (fingertip) to the lateral being more sensitive (more proximal sites). At the fingerbase, we found significant anisotropy between the proximal and lateral orientations, as well as between the lateral and oblique orientations. These results support the notion that both the GR/OR and GAP tasks are tapping a similar underlying neural mechanism.

\section{EXPERIMENT 4}

Tactile anisotropy is generally revealed in spatial measures of tactile sensitivity. Previous studies (Essock et al., 1992, 1997; Geldard \& Sherrick, 1983; B. G. Green, 1982; M. B. Jones \& Vierck, 1973; Lechelt, 1988, 1992; Vierordt, 1870; Weber, 1834/1996; Wheat \& Goodwin, 2000; Wong et al., 1974), as well as the results from Experiments 2 and 3, support this view. One could imagine, however, that other dimensions of tactile sensitivity, such as intensive or temporal dimensions, might also show anisotropy. The intensive dimension in particular is known to vary with gap width; that is, as the gap width gets smaller, the overall rate of activity in the afferent fibers decreases (Phillips \& Johnson, 1981). A task that is thought to be primarily intensive in nature but lends itself to examining differences due to orientation is the SM/GV task (Gibson \& Craig, 2002; Johnson \& Phillips, 1981). A number of other measures are also intensive in nature, such as changes in perceived magnitude or difference thresholds for intensity, but these measures lack an obvious orientation component, making them less useful in studying anisotropy.

Table 4

Grating Orientation Thresholds (in Millimeters) $\mathbf{7 5 \%}$ Correct Performance

\begin{tabular}{lccc}
\hline & Proximal-Distal & Lateral-Medial & Oblique \\
\hline Fingerpad & 1.55 & 1.23 & 1.83 \\
Fingerbase & 4.46 & 3.32 & 4.97 \\
\hline
\end{tabular}

In the $\mathrm{SM} / \mathrm{GV}$ task, subjects discriminate between a smooth contactor and one that has grooves cut into it. Similar to the GR/OR task, performance on the SM/GV task generally improves as groove width increases; however, unlike the GR/OR task, performance on the SM/GV task remains the same when sites are tested that show moderate differences in the density of innervation. With large changes in the density of innervation, for example, between the fingerpad and palm, SM/GV sensitivity changes relatively little compared to the large changes in GR/OR sensitivity (Gibson \& Craig, 2002). For this and other reasons, the $\mathrm{SM} / \mathrm{GV}$ task is not considered a measure of spatial sensitivity. In an earlier study, Essock et al. (1997) used a version of the SM/GV task to assess tactile anisotropy on the distal fingerpad. In their study, they tested sensitivity in three orientations on the distal fingerpad - proximal, lateral, and oblique. They observed significant anisotropy on the fingerpad, with the lateral orientation being the most sensitive and the proximal orientation (the orientations as defined in the present article) the least sensitive of the three orientations. From Essock's results one might expect to find significant anisotropy at other body sites using the SM/GV task. A later study, however, was unable to replicate Essock's findings, leaving unclear the role of the SM/GV task in revealing anisotropy (Craig, 1999).

In Experiment 4, the SM/GV task was used to assess anisotropy at four locations: the fingertip, fingerpad, fingerbase, and forearm. We reasoned that if the $\mathrm{SM} / \mathrm{GV}$ task relied primarily on intensive rather than spatial mechanisms, there should be little or no evidence of anisotropy with this measure. Such a finding would lend support to the view that anisotropy is the result of differences in the processing of spatial information.

\section{Method}

Subjects. For the fingertip and fingerbase, 7 subjects were tested ( 2 males, 5 females, fingertip; 4 males, 3 females, fingerbase). For the fingerpad and forearm, 9 different subjects were tested ( 5 males, 4 females).

Stimuli. The same domed grooved contactors that were used in Experiment 3 were used in Experiment 4. A smooth contactor (no grooves) was also used. The smooth contactor was identical to the grooved contactors, except that it lacked grooves. When the test site was the forearm, a set of larger domed contactors were used. These contactors were $50 \mathrm{~mm}$ in diameter (Table 1).

Apparatus. When the testing sites were on the hand, the leverarm apparatus (Experiments 2 and 3) was used. On the forearm, a different apparatus was used. This apparatus allowed the contactor to be lowered vertically onto the skin with a controlled force of $100 \mathrm{~g}$.

Procedure. Each subject was tested over eight sessions (four lateral, four proximal) for the fingertip and fingerbase and six sessions (three lateral, three proximal) for the fingerpad and forearm. The testing procedures were the same as those used in our previous experiments. At the beginning of the session, the subjects were instructed that one of two contactors would be presented (smooth or grooved). On each trial, the subject was to respond with either "smooth" or "grooved." Each session began with practice trials during which feedback was provided. During testing, however, no feedback was provided. To reduce the possibility that thermal cues might aid in discriminating between the smooth and the grooved contac- 


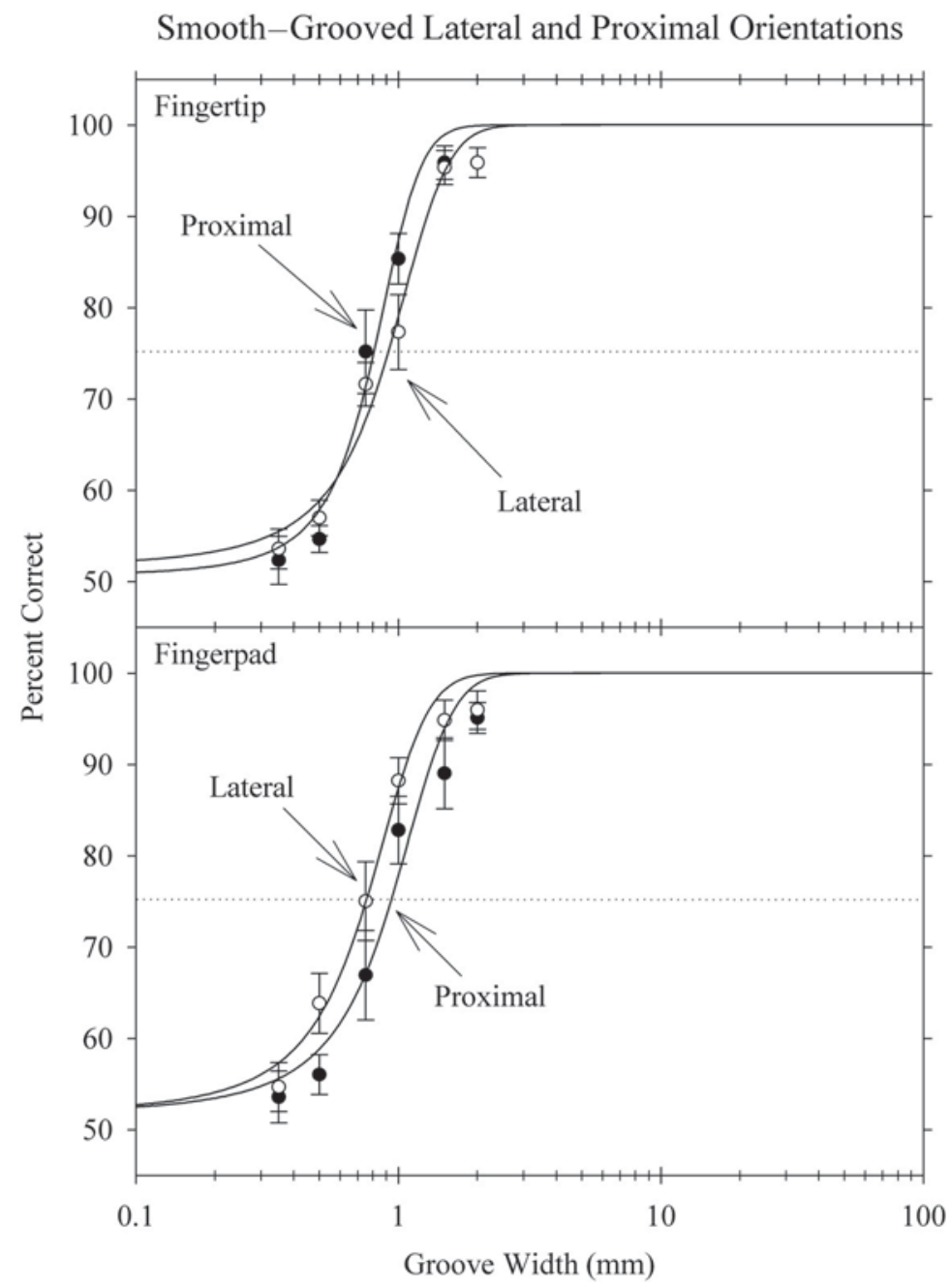

Figure 6. Experiment 4. Percentage correct as a function of log groove width (in millimeters) at the fingertip, fingerpad, fingerbase, and forearm for two orientations (proximal and lateral) of the contactor for the smooth-grooved task. Error bars represent \pm 1 standard error of the mean. The dashed line represents $\mathbf{7 5 \%}$ correct performance.

tors, the contactors were warmed to approximate skin temperature (Craig, 1999). A session consisted of six blocks of 30 trials, for a total of 180 trials. For all three test sites, testing began with the largest groove width. Successive blocks of trials tested smaller groove widths in order (Table 1).

\section{Results and Discussion}

The psychometric functions obtained for the SM/GV task in the two orientations are shown in Figure 6. The percentage correct was plotted as a function of the log of the groove width. It appears that changing orientation in the $\mathrm{SM} / \mathrm{GV}$ task produces a much smaller change in performance than changing orientation in either the GAP or GR/OR tasks. As in the previous experiments, the data were fitted with a four-parameter, sigmoid function (Equation 1). For each of the locations a repeated mea- sures ANOVA was conducted to see if there was a significant difference in performance between the two orientations (proximal vs. lateral). There was no significant difference between the lateral and proximal orientations at any of the locations except the fingerpad. Table 5 shows the results of the ANOVA.

Thresholds were calculated using the same method as in the previous experiments, $75 \%$ correct. The thresholds for the $\mathrm{SM} / \mathrm{GV}$ task in both orientations are presented in Table 6. In an earlier study, we examined SM/GV sensitivity in the lateral orientation (definition used in this study) at three locations on the hand (fingerpad, fingerbase, and palm). The average threshold in that study was $0.84 \mathrm{~mm}$, with a range of $0.63-0.96 \mathrm{~mm}$ (Table 2). The $\mathrm{SM} / \mathrm{GV}$ thresholds in the current study are approximately the same as those found in the previous study (Table 6). 


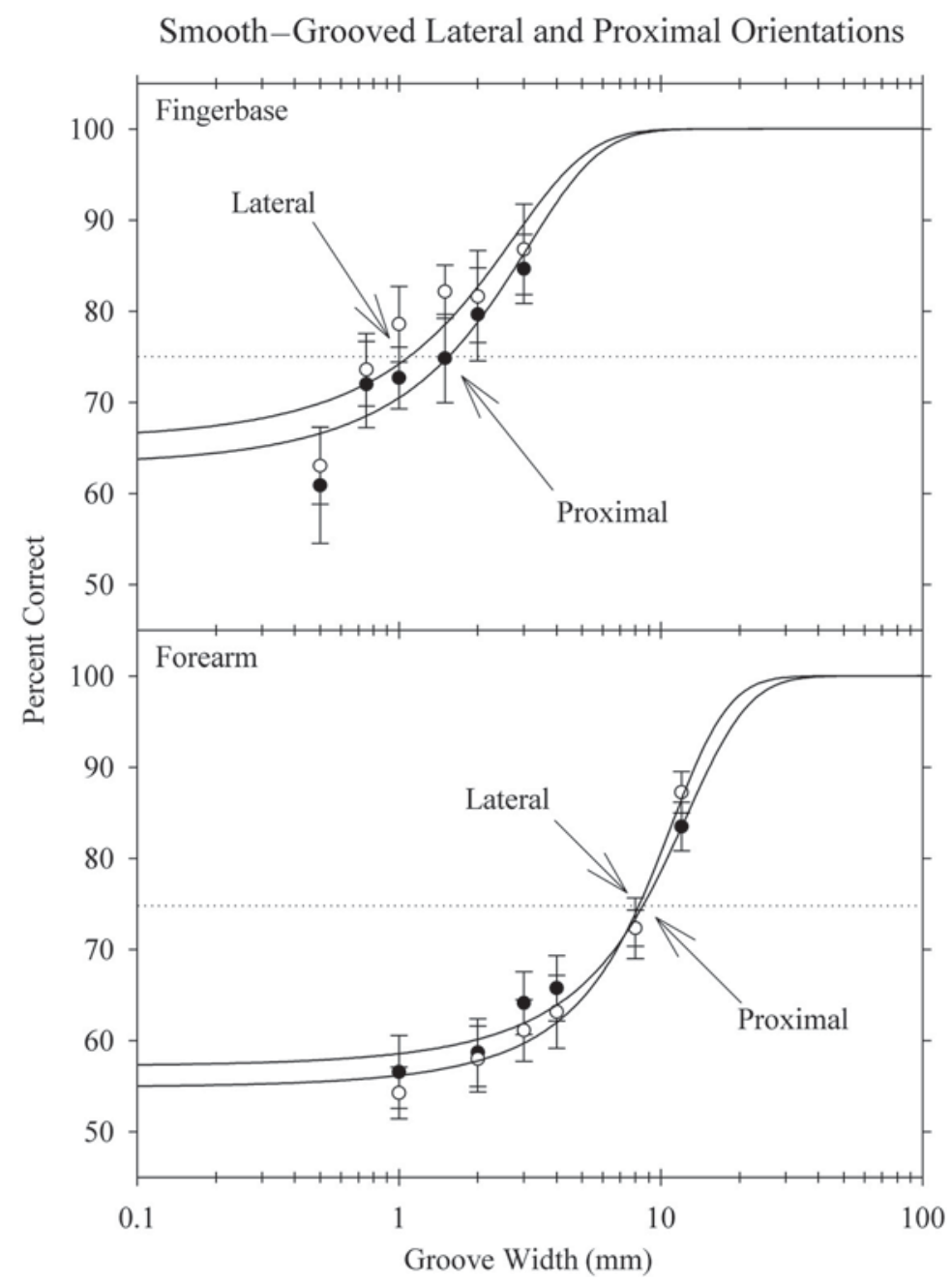

Figure 6 (Continued).

Performance on the SM/GV task changed little as different sites on the glabrous skin of the hand were tested (present study; Gibson \& Craig, 2002). At these same sites, the spacing between receptors (fingertip to palm) varied by as much as 3.5:1 (Darian-Smith \& Kenins, 1980; Johansson \& Vallbo, 1979). From these results, we concluded that the $\mathrm{SM} / \mathrm{GV}$ task was not being performed on the basis of spatial cues and was most likely being performed on the basis of intensive cues. In addition, the $\mathrm{SM} / \mathrm{GV}$ results differed from the GAP and GR/OR results in two important ways. First, as has been noted, there was

Table 5

Smooth-Grooved ANOVA for the Main Effect of Orientation

\begin{tabular}{lccl}
\hline Location & $d f$ & $F$ & $p$ \\
\hline Fingertip & 1,7 & 3.36 & .11 \\
Fingerpad* & 1,8 & 7.67 & .02 \\
Fingerbase & 1,6 & 1.41 & .28 \\
Forearm & 1,8 & 0.16 & .70 \\
\hline
\end{tabular}

*Denotes a significant main effect of orientation. a much smaller change in threshold with location. The exception was the forearm, where threshold increased by a factor of 10 as compared with the fingertip and fingerpad (Table 6). The thresholds on the forearm, however, were still below those found with the GAP task on the forearm. Second, unlike the GAP and GR/OR tasks, the orientation of the stimulus in the $\mathrm{SM} / \mathrm{GV}$ task did not produce large changes in performance.

One reason the $\mathrm{SM} / \mathrm{GV}$ thresholds may have been higher on the forearm than expected is the force used to present the contactors to the skin. With the smaller contactors (i.e., $19 \mathrm{~mm}$ in diameter), we used $100 \mathrm{~g}$ of force. This same force was used in the presentation of the larger contactors (i.e., $50 \mathrm{~mm}$ in diameter). Increasing the surface area of the contactor, while holding force constant, results in less conformity of the skin to the edges of the grooves. The curvature of the skin to an edge is positively correlated with the response rate of the primary afferent fibers (LaMotte \& Srinivasan, 1987a, 1987b). In some additional observations, higher forces were used, $400 \mathrm{~g}$ 
Table 6

Smooth-Grooved Thresholds (in Millimeters): 75\% Correct Performance Proximal-Distal Versus Lateral-Medial and Proximal to Lateral Ratio

\begin{tabular}{lccc}
\hline & Proximal-Distal & Lateral-Medial & Ratio \\
\hline Fingertip & 0.80 & 0.91 & $0.87: 1$ \\
Fingerpad & 0.94 & 0.75 & $1.25: 1$ \\
Fingerbase & 1.54 & 1.09 & $1.41: 1$ \\
Forearm & 8.62 & 8.30 & $1.03: 1$ \\
\hline
\end{tabular}

of force, and the threshold dropped from $8.62 \mathrm{~mm}$ to $3.50 \mathrm{~mm}$. In addition, the large increase in the SM/GV threshold on the forearm is undoubtedly a reflection of the large change in the density of innervation (Vallbo, Olausson, Wessberg, \& Kakuda, 1995), although this may not necessarily mean that the basis for the discrimination has shifted from primarily intensive to primarily spatial mechanisms. At less densely innervated sites like the palm and forearm, grooves of greater width may be needed before a sufficient number of receptors are recruited to differentiate between the overall neural response to a smooth surface and the response to a grooved surface. The task is still done on the basis of intensive information, that is, differences in the overall rate of firing. This view is critical for the issue of what is meant by spatial and intensive coding and what the relationship is between the two. If we define these terms in relation to the stimulus, then in the $\mathrm{SM} / \mathrm{GV}$ task we have a spatial stimulus; that is, performance varies with the spatial dimension of the stimulus, groove width. If, however, we define spatial and intensive in relation to the neural code, then the SM/GV task may rely on an intensive neural code - that is, performance varies as the level of activity in the peripheral afferent fibers varies. As has been pointed out, there is not necessarily a one-to-one relationship between the stimulus dimensions being manipulated and the underlying neural code (Johnson \& Phillips, 1984).

Even though the SM/GV task in most cases did not reveal significant levels of anisotropy, the direction of anisotropy was always consistent with the direction found with GAP and GR/OR tasks. There are parallels between the ratios obtained with the SM/GV task (Table 6), proximal-distal to lateral-medial, and the ratios obtained with the GAP task (Table 3). With both tasks, the ratio at the fingertip favored the proximal-distal orientation, whereas the lateralmedial orientation tended to be favored at the more proximal sites. What might account for this relationship leads to a discussion of the mechanisms underlying anisotropy, which will be considered in the General Discussion.

\section{GENERAL DISCUSSION}

\section{Gap Detection as a Measure of Spatial Acuity}

We have concluded that the GAP task is a suitable measure of spatial sensitivity based on the following four observations:
1. Changes in sensitivity are correlated with changes in the density of innervation. The GAP thresholds were lowest at the fingertip and highest on the forearm and upper arm. The GAP thresholds increased from the distal to the proximal locations in a manner roughly correlated with the measured density of innervation (Darian-Smith \& Kenins, 1980; Johansson \& Vallbo, 1979; Vallbo et al., 1995). This is the same pattern of responses seen with the GR/OR task (Gibson \& Craig, 2002).

2. In Experiment 1, we found that with the GAP task the psychometric functions obtained across a wide variety of locations had generally similar slopes when the results were plotted on a log plot. This similarity indicates that sensitivity is changing by a multiplicative factor. This is the predicted result for psychophysical tasks engaging spatial mechanisms (Loomis \& Lederman, 1986) and is the same result found with the GR/OR task (Craig, 1999; Craig \& Lyle, 2002; Gibson \& Craig, 2002; Johnson \& Phillips, 1981; Van Boven \& Johnson, 1994a).

3. With the exception of the palm, the glove had a modest effect on the GAP task, similar to the effect that gloves had on the GR/OR task and unlike the effect that it had on the $S M / G V$ task. These results led us to conclude that the GAP task was likely being performed using the same spatial mechanism as the GR/OR task.

4. The GAP task can be used on a wide range of locations and sensitivities. As previously discussed, one of the limitations of the GR/OR task is that it can be used on only relatively sensitive areas of the body and with subjects who have good sensitivity (Patel et al., 1997; Stevens \& Choo, 1996). In previous work, Stevens and coworkers (Stevens et al., 2003; Stevens \& Choo, 1996; Stevens \& Patterson, 1995) used the GAP task to test a large number of sites on the body and with subjects of various ages. The results of Experiments 1 and 2 are consistent with those previously obtained by Stevens et al.

Throughout this article, we have stated that performance on spatial tasks should vary across locations in a manner correlated with the density of innervation of the primary afferent fibers, specifically SAIs. From neurophysiological data (Johansson \& Vallbo, 1979; Vallbo et al., 1995), the spacing between SAI receptors can be estimated to be $1.2 \mathrm{~mm}$ for the fingertip, $1.8 \mathrm{~mm}$ for the fingerbase, $3.5 \mathrm{~mm}$ for the palm (Craig \& Lyle, 2002; Gibson \& Craig, 2002; Johansson \& Vallbo, 1983), and $5 \mathrm{~mm}$ for the forearm (Vallbo et al., 1995). From these estimates, one can calculate the expected ratios of sensitivity for the fingertip to the fingerbase, to the palm, and to the forearm to be $1.5: 1,2.9: 1$, and 4.2:1. The observed ratios of sensitivity for the fingertip to fingerbase, palm, and forearm calculated from the average GAP threshold values $(1.35-\mathrm{mm}$ tip, $4.83-\mathrm{mm}$ base, $5.77-\mathrm{mm}$ palm, and 15.34-mm forearm) in Tables 2 and 3 were 3.6:1, 4.3:1, and 11.4:1.

Although correlated with the spacing between receptors, performance for both the GR/OR and GAP tasks showed poorer sensitivity than predicted on all sites other 
than the fingertip. One possibility is that the estimates of the density of innervation were inaccurate. The estimates were based on a relatively small number of fiber samples. Craig and Lyle (2001), however, have suggested that at locations where sensitivity is poorer than predicted by the spacing between receptors, performance may be further limited by central mechanisms. The fingertip's greater use in sensory discriminations may account in part for its showing higher sensitivity, relative to spacing between receptors, than other areas of the hand (Craig \& Lyle, 2002). It may be that the density of innervation should be thought of as one of several factors limiting spatial sensitivity rather than as the sole limiting factor.

\section{Spatial Measures of Anisotropy}

The results from Experiments 2 and 3 lead to three main observations:

1.The direction of anisotropy is inconsistent across location. At the fingertip, the proximal orientation was the most sensitive. At the other locations where anisotropy was found, the lateral orientation was the most sensitive.

2 . The amount of anisotropy varied by location. As revealed by the ratio between thresholds in the two orientations, the amount of anisotropy varied from essentially 0 , a $1: 1$ ratio (fingerpad), to a ratio of 2.35:1 (forearm). However, with the exception of the forearm, the five sites that showed anisotropy-fingertip, middle pad, fingerbase, palm, and upper arm - showed reasonably similar ratios. The fingertip ratio (lateral-medial to proximal-distal) was $1.49: 1$, with remaining sites ranging from $1.35: 1$ to $1.64: 1$. The amount of anisotropy on the forearm is strikingly different from the other locations tested. At present, we have no explanation for this difference.

3. As suggested by the second point above, there is no simple relationship between sites of stimulation and the amount of anisotropy. Clearly, differences in spatial sensitivity depending upon orientation need to be taken into account when spatial sensitivity is measured at different sites.

In the tactile system, several factors have been proposed to account for anisotropy. In general, these factors can be grouped into mechanical (skin ridges and tissue differences), peripheral neural (receptive field shape and the distribution of receptors), and central neural (the number of orientation-selective neurons and distorted cortical representations). The present results do not support the position that any of these mechanisms are the sole cause of anisotropy in the somatosensory system.

As noted, other investigators have found improved performance when the edges of grooved contactors were parallel rather than perpendicular to skin ridges (VegaBermudez \& Johnson, 2004; Wheat \& Goodwin, 2000). We also found greater sensitivity (at the fingertip) when the edges of the gap were oriented parallel to the skin ridges. Skin ridges may be one of the causes of anisotropy on the fingertip, but they cannot be responsible for anisotropy on locations without skin ridges, such as the forearm and upper arm.
At least two possible peripheral neural mechanisms could affect tactile anisotropy. If the receptive fields were elongated along the proximal-distal axis of the arm and hand, they could be the mechanism that produces anisotropy (Stevens \& Patterson, 1995; Weber, 1834/1996). If the same size gap were placed across the arm, it would be more likely to stimulate neurons with narrower receptive fields than when it is placed along the arm where the receptive fields would be elongated. Some nonhuman primate studies have looked at the size and shape of the peripheral afferent fibers' receptive fields. In general these studies have found that the SAI afferent fibers' receptive fields increase in size with decreasing density of innervation, have sharp delimited boundaries that change little with increasing force, and can be round to oval in shape (Vallbo et al., 1995). No study that we know of, however, has reported a systematic bias in the shape and orientation of the peripheral afferent fibers' receptive fields that would account for the observed anisotropy.

The second possible peripheral neural mechanism is the spacing between receptors. If receptors were spaced regularly and consistently closer to one another in one orientation compared with the other orientation, differences in spatial acuity might be expected. This mechanism is particularly appealing because performance on both the GR/OR and GAP tasks decreases with increasing spacing between the peripheral afferent fibers. Currently the exact spacing between Merkel receptors, the receptors associated with the SAI fibers, is unexplored. However, the mappings of receptive fields for SAI fibers (Vallbo et al., 1995) show neither the regularity nor difference in spacing that might account for anisotropy.

Central mechanisms are also possible mechanisms for tactile anisotropy. In vision, for example, a central mechanism is thought to be responsible for anisotropy (De Valois, Yund, \& Hepler, 1982; Leventhal \& Hirsch, 1977; Mansfield, 1974; Mansfield \& Ronner, 1978; Orban \& Kennedy, 1981). In visual anisotropy, greater sensitivity is found for vertical and horizontal orientations than for the oblique orientation. This is believed to be due to a greater number of orientation-selective neurons in the visual cortex that are sensitive to vertical and horizontal bars relative to those that are sensitive to oblique bars (Appelle, 1972). It is possible that in the tactile system, a greater number of orientation-selective neurons are sensitive to edges oriented along the proximal-distal axis, and in fact, there is evidence to suggest that is the case (Essock et al., 1992; Hsiao, Lane, \& Fitzgerald, 2002).

A neurophysiological explanation is complicated by the fact that anisotropy is not uniform along the finger and palm. There is a reversal as one moves from the fingertip to the fingerbase and palm (see Figure 4). If anisotropy were due to central mechanisms, then from the present results, one would expect to be able to find substantial differences in the representation of spatial information in the somatosensory cortex depending on the peripheral location being represented. The detailed neurophysiologi- 
cal work that might reveal such differences, however, is not yet available.

An additional factor that might promote anisotropy is the anatomy and use of the particular sites of stimulation. Neurophysiological data indicate that when simultaneous inputs are presented to areas of skin, receptive field sizes tend to increase and represent more disparate areas (Clark, Allard, Jenkins, \& Merzenich, 1988). From the fingerpad to more proximal sites, greater sensitivity is found in the lateral-medial orientation. Sensitivity might be less in the proximal-distal orientation because, in the course of use, more widely separated sites on this axis are contacted simultaneously than sites in the lateral-medial orientation. For example, when the forearm rests on a surface, considerably more of the limb in the proximal-distal orientation contacts the surface than in the lateral-medial orientation.

The last two possible mechanisms of anisotropy to be discussed are the effects of lateral inhibition and dermatomes. Lateral inhibition has been suggested previously as a possible mechanism of anisotropy (Fuchs \& Brown, 1984). For lateral inhibition to contribute to anisotropy, there would need to be greater lateral inhibition in the orientation of greater sensitivity, thereby sharpening the representation of the edge. In discussing their two-point discrimination data, Fuchs and Brown noted that inhibiting interneurons may align perpendicular to the long axis of the limbs (E. G. Jones, 1981). This orientation might help account for finding greater sensitivity across rather than along the limb (Fuchs \& Brown, 1984). The final mechanism of action is that of dermatomal boundaries (Geldard, 1975; Lee, McGillis, \& Greenspan, 1996). The idea is that stimuli that cross dermatomal boundaries are more easily distinguished than are stimuli that are presented within a dermatome. Dermatomes on the forearm, for example, have an elongated representation, which might contribute to the large anisotropic effect. Dermatomal representation, however, cannot be the mechanism of action on the index finger and thenar eminence because one dermatome encompasses that whole region of the hand (Keegan \& Garrett, 1948).

In conclusion, spatial anisotropy was found at all locations that were tested, except the fingerpad. As one moves from the most distal location to more proximal locations on the finger, the direction of anisotropy reverses, with no anisotropy evident on the center of the fingerpad. The greatest amount of anisotropy was seen on the forearm. These results suggest that whatever the underlying causes of anisotropy, these causes should be large and change with location. Furthermore, for a particular location, different factors or combinations of factors are likely to be responsible for anisotropy, and such factors vary with location. The results with the SM/GV task, likely done on the basis of intensive information, suggest that tactile anisotropy is limited to spatial stimuli. Finally, our findings suggest a need for neurophysiological measurements to be made at different locations so the underlying mechanisms of anisotropy can be better understood.

\section{REFERENCES}

Appelle, S. (1972). Perception and discrimination as a function of stimulus orientation: The "oblique effect" in man and animals. Psychological Bulletin, 78, 266-278

BorING, E. G. (1942). Sensation and perception in the history of experimental psychology. New York: Appleton-Century-Crofts.

Clark, S. A., Allard, T., Jenkins, W. M., \& Merzenich, M. M. (1988). Receptive fields in the body-surface map in adult cortex defined by temporally correlated inputs. Nature, 332, 444-445.

CraIG, J. C. (1999). Grating orientation as a measure of tactile spatial acuity. Somatosensory \& Motor Research, 16, 197-206.

Craig, J. C., \& Johnson, K. O. (2000). The two-point threshold: Not a measure of tactile spatial resolution. Current Directions in Psychological Science, 9, 29-32.

Craig, J. C., \& Kisner, J. M. (1998). Factors affecting tactile spatial acuity. Somatosensory \& Motor Research, 15, 29-45.

Craig, J. C., \& Lyle, K. B. (2001). A comparison of tactile spatial sensitivity on the palm and fingerpad. Perception \& Psychophysics, 63, 337-347.

Craig, J. C., \& Lyle, K. B. (2002). A correction and a comment on Craig and Lyle (2001). Perception \& Psychophysics, 64, 504-506.

Darian-Smith, I., \& Kenins, P. (1980). Innervation density of mechanoreceptive fibers supplying glabrous skin of the monkey's index finger. Journal of Physiology, 309, 147-155.

De Valois, R. L., Yund, E. W., \& Hepler, N. (1982). The orientation and direction selectivity of cells in macaque visual cortex. Vision Research, 22, 531-544.

Essock, E. A., Krebs, W. K., \& Prather, J. R. (1992). An anisotropy of human tactile sensitivity and its relation to the visual oblique effect. Experimental Brain Research, 91, 520-524.

Essock, E. A., Krebs, W. K., \& Prather, J. R. (1997). Superior sensitivity for tactile stimuli oriented proximally-distally on the finger: Implications for mixed Class 1 and Class 2 anisotropies. Journal of Experimental Psychology: Human Perception \& Performance, 23, 515-527.

FriEdLINE, C. L. (1918). The discrimination of cutaneous patterns below the two-point limen. American Journal of Psychology, 29, 400-419.

FuCHS, J. L., \& Brown, P. B. (1984). Two-point discriminability: Relation to properties of the somatosensory system. Somatosensory \& Motor Research, 2, 163-169.

GELDARD, F. A. (1975). Sensory saltation metastability in the perceptual world. Hillsdale, NJ: Erlbaum.

Geldard, F. A., \& Sherrick, C. E. (1983). The cutaneous saltatory area and presumed neural basis. Perception \& Psychophysics, 33, 299-304.

Gibson, G. O., \& Craig, J. C. (2002). Relative roles of spatial and intensive cues in the discrimination of spatial tactile stimuli. Perception \& Psychophysics, 64, 1095-1107.

Green, B. G. (1982). The perception of distance and location for dual tactile pressures. Perception \& Psychophysics, 31, 315-323.

Green, D. M., \& Swets, J. A. (1966). Signal detection theory and psychophysics. New York: Wiley.

Greenspan, J. D., \& Bolanowski, S. J. (1996). The psychophysics of tactile perception and its peripheral physiological basis. In L. Kruger (Ed.), Pain and touch (pp. 25-103). San Diego: Academic Press.

Hsiao, S. S., Lane, J., \& Fitzgerald, P. (2002). Representation of orientation in the somatosensory system. Behavioural Brain Research, 135, 93-103.

Johansson, R. S., \& Vallbo, Å. B. (1979). Tactile sensibility in the human hand: Relative and absolute densities of four types of mechanoreceptive units in glabrous skin. Journal of Physiology, 286, 283-300.

Johansson, R. S., \& VAllbo, A. B. (1983). Tactile sensory coding in the glabrous skin of the human hand. Trends in Neurosciences, 6, 27-32.

Johnson, K. O., \& HsiaO, S. S. (1992). Neural mechanisms of tactual form and texture perception. Annual Review of Neuroscience, 15, 227-250.

Johnson, K. O., \& Phillips, J. R. (1981). Tactile spatial resolution: I. Two-point discrimination, gap detection, grating resolution, and letter recognition. Journal of Neurophysiology, 46, 1177-1191.

Johnson, K. O., \& Phillips, J. R. (1984). Spatial and nonspatial neural mechanisms underlying tactile spatial discrimination. In $\mathrm{C}$. von Euler, O. Franzén, U. Lindblom, \& D. Ottoson (Eds.), Wenner-Gren 
International Symposium Series: Vol. 41. Somatosensory mechanisms (pp. 237-248). London: Macmillan.

Johnson, K. O., Van Boven, R. W., \& Hsiao, S. S. (1994). The perception of two points is not the spatial resolution threshold. In J. Boivie, P. Hansson, \& U. Lindblom (Eds.), Touch, temperature, and pain in health and disease: mechanisms and assessments (11th ed., pp. 389404). Seattle: IASP Press.

JonEs, E. G. (1981). Anatomy of cerebral cortex: Columnar input-output organization. In F. O. Schmidt, F. G. Worden, G. Adelman, \& S. G. Dennis (Eds.), The organization of the cerebral cortex (pp. 199-235). Cambridge, MA: MIT Press.

Jones, M. B., \& VIERCK, C. J. (1973). Length discrimination on the skin. American Journal of Psychology, 86, 49-60.

Jones, M. B., ViercK, C. J., \& Graham, R. B. (1973). Line-gap discrimination of the skin. Perceptual \& Motor Skills, 36, 563-570.

KeEgan, J. J., \& GarretT, F. D. (1948). The segmental distribution of the cutaneous nerves in the limb of man. Anatomical Record, 102, 409-437.

LaMotte, R. H., \& Srinivasan, M. A. (1987a). Tactile discrimination of shape: Responses of rapidly adapting mechanoreceptive afferents to a step stroked across the monkey fingerpad. Journal of Neuroscience, 7, 1672-1681.

LaMotte, R. H., \& Srinivasan, M. A. (1987b). Tactile discrimination of shape: Responses of slowly adapting mechanoreceptive afferents to a step stroked across the monkey fingerpad. Journal of Neuroscience, 7, 1655-1671.

LECHELT, E. C. (1988). Spatial asymmetries in tactile discrimination of line orientation: A comparison of the sighted, visually impaired, and blind. Perception, 17, 579-585.

LeCHeLt, E. C. (1992). Tactile spatial anisotropy with static stimulation. Bulletin of the Psychonomic Society, 30, 140-142.

Lee, D. K., McGillis, L. B., \& Greenspan, J. D. (1996). Somatotopic localization of thermal stimuli I: A comparison of within- versus across-dermatomal separation of innocuous thermal stimuli. Somatosensory \& Motor Research, 13, 67-71.

Leventhal, A. G., \& Hirsch, H. V. (1977). Effects of early experience upon orientation sensitivity and binocularity of neurons in visua cortex of cats. Proceedings of the National Academy of Sciences, 74 1272-1276.

Loomis, J. M., \& Lederman, S. J. (1986). Tactual perception. In K. R. Boff, L. Kaufman, \& J. P. Thomas (Eds.), Handbook of perception and human performance (chap. 31, pp. 1-41). New York: Wiley.

MANSFIELD, R. J. (1974). Neural basis of orientation perception in primate vision. Science, 186, 1133-1135.

Mansfield, R. J., \& Ronner, S. F. (1978). Orientation anisotropy in monkey visual cortex. Brain Research, 149, 229-234.

Orban, G. A., \& Kennedy, H. (1981). The influence of eccentricity on receptive field types and orientation selectivity in areas 17 and 18 of the cat. Brain Research, 208, 203-208.
Patel, J., Essick, G. K., \& Kelly, D. G. (1997). Utility of square-wave gratings to assess perioral spatial acuity. Journal of Oral \& Maxillofacial Surgery, 55, 593-601.

Phillips, J. R., \& Johnson, K. O. (1981). Tactile spatial resolution: II. Neural representation of bars, edges, and gratings in monkey primary afferents. Journal of Neurophysiology, 46, 1192-1203.

Sathian, K., \& Zangaladze, A. (1996). Tactile spatial acuity at the human fingertip and lip: Bilateral symmetry and interdigit variability. Neurology, 46, 1464-1466.

Stevens, J. C., Alvarez-Reeves, M., Dipietro, L., Mack, G. W., \& Green, B. G. (2003). Decline of tactile acuity in aging: A study of body site, blood flow, and lifetime habits of smoking and physical activity. Somatosensory \& Motor Research, 20, 271-279.

Stevens, J. C., \& CHoo, K. K. (1996). Spatial acuity of the body surface over the life span. Somatosensory \& Motor Research, 13, 153-166.

Stevens, J. C., \& Patterson, M. Q. (1995). Dimensions of spatial acuity in the touch sense: Changes over the life span. Somatosensory \& Motor Research, 12, 29-47.

TAWNEY, G. (1895). The perception of two points not the space-threshold. Psychological Review, 2, 585-593.

Vallbo, Å. B., Olausson, H., Wessberg, J., \& KaKuda, N. (1995) Receptive field characteristics of tactile units with myelinated afferents in hairy skin of human subjects. Journal of Physiology, $\mathbf{4 8 3}$, 783-795.

Van Boven, R. W., \& Johnson, K. O. (1994a). The limit of tactile spatial resolution in humans: Grating orientation discrimination at the lip, tongue and finger. Neurology, 44, 2361-2366.

VAN Boven, R. W., \& Johnson, K. O. (1994b). A psychophysical study of the mechanisms of sensory recovery following nerve injury in humans. Brain, 117, 149-167

Vega-Bermudez, F., \& Johnson, K. O. (2004). Fingertip skin conformance accounts, in part, for differences in tactile spatial acuity in young subjects, but not for the decline in spatial acuity with aging. Perception \& Psychophysics, 66, 60-67.

Vierordt, K. (1870). Die Abhängigkeit der Ausbildung des Raumsinnes der Haut von den Beweglichkeit der Kerpertheile. Zeitschrift für Biologie, 6, 53-72.

Weber, E. H. (1996). E. H. Weber on the tactile senses (2nd ed.). Hove, U.K.: Erlbaum. (Original work published in 1834)

WEINSTEIN, S. (1968). Intensive and extensive aspects of tactile sensitivity as a function of body part, sex and laterality. In D. R. Kenshalo (Ed.), The skin senses (pp. 195-222). Springfield, IL: C. C. Thomas.

WheAt, H. E., \& GoodwIN, A. W. (2000). Tactile discrimination of gaps by slowly adapting afferents: Effects of population parameters and anisotropy in the fingerpad. Journal of Neurophysiology, 84, 14301444

Wong, T. S., Ho, R., \& Ho, J. (1974). Influence of shape of receptor organ on the horizontal-vertical illusion in passive touch. Journal of Experimental Psychology, 103, 414-419. 


\section{APPENDIX}

Figure A1 shows the gap stimulators and the labels, "proximal" and "lateral," that were used in the present study. When the gap detection task is compared with the grating orientation task, the issue of how to specify the orientation of the grating becomes important. In other studies (Vega-Bermudez \& Johnson, 2004; Wheat \& Goodwin, 2000), when the grooves of the gratings were aligned with the long axis of the finger, the grating was said to be in the proximal-distal orientation. However, as Figure A1 shows, grooves (nominally in a proximal-distal orientation) can be viewed as being created by a series of gaps with the gap being generated by an edge oriented in the lateral-medial orientation. As the groove widths become wider, the "gap" becomes greater in the lateral orientation. In this article and for the purposes of comparing the gap detection and grating orientation tasks, the orientation of the contactors will be defined as indicated in Figure A1. In addition, this definition is consistent with earlier studies and two-point threshold measurements.

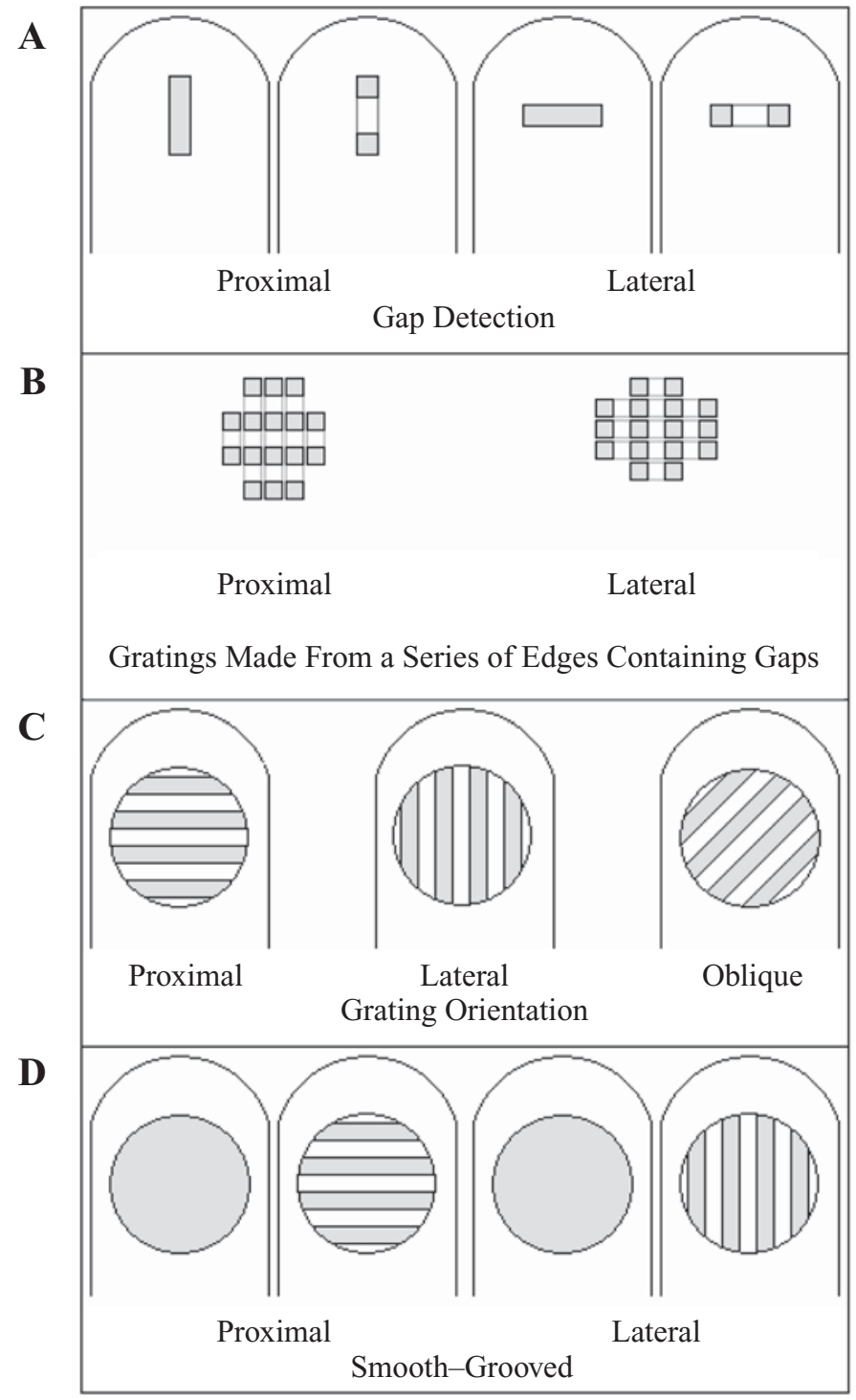

Figure A1. Schematics of the position of the contactor, its orientation on the skin, and the labeling used for all of the stimuli used in the study. Panel A shows the positioning and orientation of the edge in the gap detection task. Note that the labeling refers to orientation of the longer dimension of the contactor, the edge, and not the gap in the edge. Panel B shows how a series of edges that contain gaps might be arranged to produce a grating. Panels $C$ and $D$ show the positioning of the gratings in the grating orientation and smooth-grooved tasks. The orientations for the grating orientation and smooth-grooved tasks are labeled so that they are consistent with the gap detection task.

(Manuscript received June 3, 2004;

revision accepted for publication December 6, 2004.) 